Dipartimento di Elettronica e Informazione e Bioingegneria

Politecnico

20133 Milano (Italia)

Piazza Leonardo da Vinci, 32

di Milano

Tel. (39) 02-2399.3400

Fax (39) 02-2399.7680

\title{
Uniform Linear Arrays of First-Order Steerable Differential Microphones
}

F. Borra, A. Bernardini, F. Antonacci and A. Sarti, "Uniform Linear Arrays of First-Order Steerable Differential Microphones," in IEEE/ACM Transactions on Audio, Speech, and Language Processing, doi: 10.1109/TASLP.2019.2934567

\section{Published in:}

IEEE/ACM Transactions on Audio, Speech, and Language Processing

\section{Document Version:}

Peer reviewed version

\section{Publisher rights:}

(C) 2019 IEEE. This work is made available online in accordance with the publisher's policies. Please refer to any applicable terms of use of the publisher. 


\title{
Uniform Linear Arrays of First-Order Steerable Differential Microphones
}

\author{
Federico Borra, Student Member, IEEE, Alberto Bernardini, Member, IEEE, \\ Fabio Antonacci, Member, IEEE, Augusto Sarti, Senior Member, IEEE
}

\begin{abstract}
We propose a spatial filtering method for linear arrays of First-Order Steerable Differential Microphones (FOSDMs), which operates in two layers. In the former, signals acquired by individual microphones are locally filtered to produce the outputs of the FOSDMs. In the latter, the outputs of the FOSDMs are processed by another filter. We analyse different design methodologies and study the conditions under which the two filtering layers can be decoupled. The proposed two-layer spatial filter can be flexibly controlled with a single scalar parameter, which can be chosen, for example, to maximize the White Noise Gain (like in a Delay-and-Sum beamformer); or to maximize the Directivity Factor (like in a Super-Directive beamformer); without needing any matrix inversion. The effectiveness of the proposed beamforming method is compared with traditional spatial filtering techniques using different metrics.
\end{abstract}

Index Terms-Beamforming, Differential Microphones

\section{INTRODUCTION}

D IFFERENTIAL Microphones (DMs) [1]-[7] have been extensively investigated in the literature on small-size microphone arrays, e.g., arrays of MEMS microphones [8], because of their ability of spatially filtering broadband audio signals, such as speech signals, with a flat response for a wide frequency range. Traditional First-Order Steerable Differential Microphones (FOSDMs) are characterized by first-order beam patterns [1], [9], built through the linear combination of first-order eigenbeams, thus enabling a simple control of the main lobe direction and of the beampattern shape. In the past few years, a great deal of effort has been spent on extending the theory of differential beamforming in order to design DMs characterized by higher order beampatterns. Early implementations of $N$ th order DMs are based on the weighted sum of derivatives of the sound pressure up to the $N$ th order, approximated through the recursive difference of the signals acquired by the individual microphones [4], [10]. This approximation is valid under the assumption that the distance between sensors is much smaller than the wavelength of the sound signal of interest. The beamforming methods in [4], [10] have been generalized to implement high-order DMs using arrays with different geometries [5]-[7], [11] and to decouple the number of sensors from the order of the target beam pattern [7], [12]-[15]. Moreover, different techniques have been developed for performing steering of high-order beams [16]-[21].

F. Borra, A. Bernardini, F. Antonacci and A. Sarti are with the Dipartimento di Elettronica, Informazione e Bioingegneria, Politecnico di Milano, 20133 Milan, Italy (e-mail: federico.borra@polimi.it; alberto.bernardini@polimi.it; fabio.antonacci@polimi.it; augusto.sarti@polimi.it).
Despite the highlighted features, DMs suffer from some problems that have been deeply investigated in the literature. In particular, they have been shown to amplify white noise, especially at low frequency [4]-[6], [10] and to exhibit high sensitivity to sensor mismatches [3], [22], [23]. The relevance of these problems grows as the order $N$ increases, which explains why most of the literature focuses on first- or secondorder DMs.

Though the literature on the design of DMs is well established, to the best of our knowledge no systematic study has been conducted on beamforming systems based on the combination of multiple DMs. As a first step in that direction, this manuscript proposes a beamforming system based on a uniform linear array of FOSDMs, and offers an extensive characterization of its features. More specifically, we consider an array of omnidirectional microphones, which are organized in subarrays of four microphones each (FOSDM), whose centers are uniformly spaced along a line. We therefore address the design of a beamformer based on an array of FOSDMs, and compare its performance to that of standard beamforming techniques applied to an array of omnidirectional sensors with the same geometry. The beamformer presented in this manuscript is based on two filtering stages; the first stage computes the output signals of FOSDMs and the second stage filters such signals. We will show that the small aperture of FOSDMs is key to decouple the design of the two aforementioned filtering stages. The two-stage system results in a tunable beamformer, whose behavior can gradually morph from that of a Delay-and-Sum beamformer [24, p. 26] to that of a Super-Directive [24, p. 28] beamformer. We present an implementation of that system, whose morphing is controlled by a single scalar parameter. Unlike traditional Super-Directive beamformers [24. p. 28], this implementation does not require any matrix inversion and bypasses numerous numerical problems that are typically associated to ill-conditioning. The proposed beamformer, based on an uniform linear array of FOSDMs, exhibits a directivity factor whose variability with frequency and steering angle is less pronounced than the one obtained with an array of omnidirectional sensors. The presented system turns out to be particularly suitable for flexible spatial filtering and signal enhancement.

Finally, it is worth mentioning that the proposed approach, though discussed only for linear arrays, can be readily extended to other geometries.

The manuscript is organized as follows. Section $\Pi$ describes the signal model of the analyzed system. Two spatial filtering approaches employing the same array geometry are discussed. 


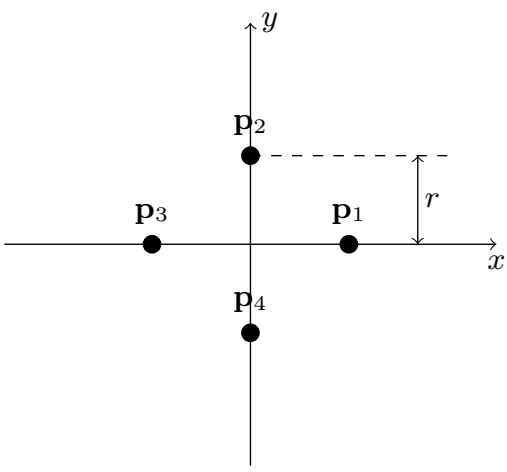

Fig. 1: The microphones configuration of a First-Order Steerable Differential Microphone (FOSDM). Four microphones are equally spaced on a circle of radius $r$. Without loss of generality the first microphone is placed on the positive $x$ axis.

The first approach is based on a direct filtering of signals acquired by omnidirectional sensors. The second approach is based on a layered structure consisting of two filtering stages; one computing the output signals of FOSDMs (local filtering) and the other processing those signals (global filtering). Section $\mathbb{m}$ also provides the definition of the beamformer performance measures. Section [II] addresses the design of the beamforming filters and, for the layered approach, shows under which conditions global filtering can be decoupled from local filtering. Section $[\mathrm{IV}$ describes how the proposed system can be tuned to behave like a Delay-And-Sum (DAS) or a SuperDirective (SD) beamformer. Section $\nabla$ shows the effectiveness of the proposed spatial filters in terms of White Noise Gain and Directivity Factor, while Section $\nabla \mathbf{V I}$ characterizes the same spatial filters with respect to a wider set of metrics. Finally, Section VII presents a straightforward implementation scheme for the proposed system and a modified, though equivalent, version which allows us to control the "tuning" between DAS and SD beamformers adjusting a single scalar parameter.

\section{Signal Model and Performance Measures}

In this section we derive the signal model of the proposed system. Since our system is composed by a set of FOSDMs, we start by deriving the signal model of a given FOSDM in the array and then we extend this model in order to derive the signal model of the entire system.

\section{A. FOSDM Signal Model}

Let us consider a system composed of $K$ FOSDMs each of which has a geometry as the one depicted in Fig. 1. The $m$ th sensor in the $k$ th array is located at $\mathbf{p}_{m}^{(\mathrm{k})}=\left[p_{m, x}^{(k)}, p_{m, y}^{(k)}\right]^{T}$. Assuming a far field hypothesis (i.e., the dimension of the array is much smaller than the wavelength), we can write the propagation vector of a $2 \mathrm{D}$ plane wave from a source to the $k$ th FOSDM as

$$
\overline{\mathbf{d}}^{(k)}(\omega, \theta)=\left[\bar{D}_{1}^{(k)}(\omega, \theta), \ldots, \bar{D}_{4}^{(k)}(\omega, \theta)\right]^{T}
$$

with

$$
\bar{D}_{m}^{(k)}(\omega, \theta)=e^{-j \frac{\omega}{c}\left(\cos (\theta) p_{m, x}^{(k)}+\sin (\theta) p_{m, y}^{(k)}\right)}, m=1, \ldots, 4,
$$

where the superscript ${ }^{T}$ denotes transposition, $j=\sqrt{-1}$ is the imaginary unit, $\omega=2 \pi f$ is the angular frequency, $f>0$ is the temporal frequency, $c$ is the speed of sound, $\theta$ is the direction of arrival of the source signal to the array $(\theta=0$ refers to the broadside direction). Given the propagation vector defined in (1), the signal at the $m$ th microphone in the $k$ th FOSDM is expressed as

$$
\bar{Y}_{m}^{(k)}(\omega)=\bar{D}_{m}^{(k)}(\omega, \theta) X(\omega)+\bar{V}_{m}^{(k)}(\omega), m=1, \ldots, 4,
$$

where $X(\omega)$ is the source signal and $\bar{V}_{m}^{(k)}(\omega)$ models an additive noise. In vectorial form, (3) can be written as

$$
\overline{\mathbf{y}}^{(k)}(\omega)=\overline{\mathbf{d}}^{(k)}(\omega, \theta) X(\omega)+\overline{\mathbf{v}}^{(k)}(\omega),
$$

where $\overline{\mathbf{y}}^{(k)}(\omega)=\left[\bar{Y}_{1}^{(k)}(\omega), \ldots, \bar{Y}_{4}^{(k)}(\omega)\right]^{T}$ and $\overline{\mathbf{v}}^{(k)}(\omega)=$ $\left[\bar{V}_{1}^{(k)}(\omega), \ldots, \bar{V}_{4}^{(k)}(\omega)\right]^{T}$.

In order to spatially filter the signal acquired by the $k$ th FOSDM, a weight $A_{m}^{*}(\omega)$ is applied to the output of each microphone, where the asterisk denotes complex conjugation. The weighted outputs are then summed to form the output signal of a FOSDM. If we collect the weights in the vector

$$
\mathbf{a}^{(k)}(\omega)=\left[A_{1}^{(k)}(\omega), \ldots, A_{4}^{(k)}(\omega)\right]^{T},
$$

we can write the output of the filter as

$$
\begin{aligned}
Z^{(k)}(\omega)= & \sum_{m=1}^{4} A_{m}^{*(k)}(\omega) \bar{Y}_{m}^{(k)}(\omega) \\
= & {\left[\mathbf{a}^{(k)}(\omega)\right]^{H} \overline{\mathbf{y}}^{(k)}(\omega) } \\
= & {\left[\mathbf{a}^{(k)}(\omega)\right]^{H} \overline{\mathbf{d}}^{(k)}(\omega, \theta) X(\omega) } \\
& +\left[\mathbf{a}^{(k)}(\omega)\right]^{H} \overline{\mathbf{v}}^{(k)}(\omega),
\end{aligned}
$$

where ${ }^{H}$ indicates Hermitian transposition. The design of the filter $\mathbf{a}^{(k)}(\omega)$ will be addressed in the Subsubsection III-B1

\section{B. Proposed System Signal Model}

In the following we show how the signal model of a single FOSDM, presented previously, can be readily extended in order to describe an uniform linear array of $K$ FOSDMs (see Fig. 2). Starting from the steering vector $\overline{\mathbf{d}}^{(k)}(\omega, \theta)$ of the $k$ th FOSDM of the array, and defined in (1), the propagation vector $\mathbf{d}(\omega, \theta) \in \mathbb{C}^{4 K \times 1}$ of the proposed system can be obtained by stacking the propagation vectors of the FOSDMs composing the array, i.e.,

$$
\begin{aligned}
\mathbf{d}(\omega, \theta) & =\left[D_{1}(\omega, \theta), \ldots, D_{4 K}(\omega, \theta)\right]^{T} \\
& =\left[\overline{\mathbf{d}}^{(1)}(\omega, \theta)^{T}, \ldots, \overline{\mathbf{d}}^{(K)}(\omega, \theta)^{T}\right]^{T} .
\end{aligned}
$$

Assuming, without loss of generality, that the array is deployed along the $y$ axis, as in Fig. 2, the position of the $m$ th sensor of the $k$ th FOSDM can be conveniently expressed as a function 


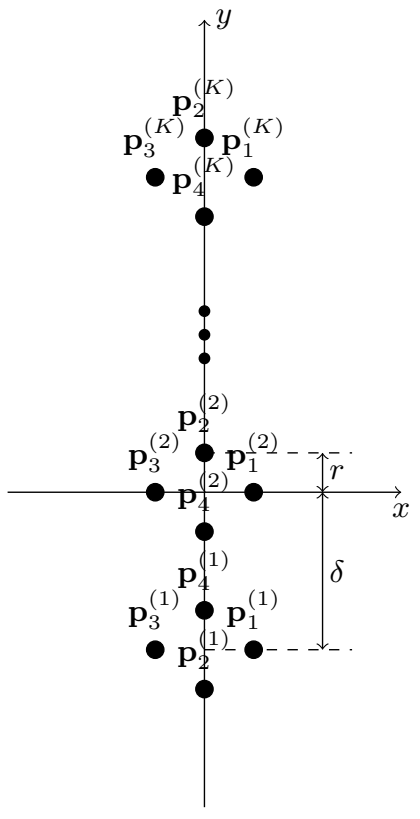

Fig. 2: Configuration of sensors in the proposed system.

of the FOSDM radius $r$ and the distance $\delta$ between two consecutive FOSDMs as

$$
\begin{aligned}
p_{m, x}^{(k)} & =r \cos \left(\frac{\pi}{2}(m-1)\right) \\
p_{m, y}^{(k)} & =r \sin \left(\frac{\pi}{2}(m-1)\right)+\delta \frac{2 k-K-1}{2} \\
m & =1, \ldots, 4 \quad k=1, \ldots, K .
\end{aligned}
$$

We finally obtain the signal model of the overall array by writing

$$
\mathbf{y}(\omega)=\mathbf{d}(\omega, \theta) X(\omega)+\mathbf{v}(\omega)
$$

In the following we do not make any explicit assumption about the distance between individual FOSDM composing the array, but we assume that the wavelength is much larger than the FOSDM radius. In the following we discuss two possible strategies to design a spatial filter for the proposed system with $4 K$ omnidirectional sensors (see Fig. 2); the former considers the system as an array of omnidirectional microphones, while the latter treats it as an uniform linear array of FOSDMs.

1) Direct filtering approach: This approach is the one that is traditionally used to spatially filter the signal acquired by a microphone array. The filtered output is given by

$$
T(\omega)=\mathbf{w}^{H}(\omega) \mathbf{y}(\omega),
$$

where $\mathbf{w}(\omega)$ is a $4 K$-tap spatial filter defined as

$$
\mathbf{w}(\omega)=\left[W_{1}(\omega), \ldots, W_{4 K}(\omega)\right]^{T} .
$$

2) Two-stage filtering approach: The second approach exploits the fact that the system is composed of FOSDMs and the output is the result of a two-stage filtering. The first stage consists of local filtering, i.e. the output signals of FOSDMs are computed. In the second stage the output signals of all the FOSDMs are properly combined to obtain the desired spatial filtering effect. Formally, we can write

$$
T(\omega)=\mathbf{h}^{H}(\omega) \mathbf{z}(\omega),
$$

where

$$
\mathbf{h}(\omega)=\left[H_{1}(\omega), \ldots, H_{K}(\omega)\right]^{T}
$$

is the filter that combines the output signals of all FOSDMs. In the following, we refer to $\mathbf{h}(\omega)$ as the global filter. The vector $\mathbf{z}(\omega)$, which contains the FOSDM output signals, is given by

$$
\begin{aligned}
\mathbf{z}(\omega) & =\left[Z^{(1)}(\omega), \ldots, Z^{(K)}(\omega)\right]^{T} \\
& =\left[\mathbf{a}^{H}(\omega) \overline{\mathbf{y}}^{(1)}(\omega), \ldots, \mathbf{a}^{H}(\omega) \overline{\mathbf{y}}^{(K)}(\omega)\right]^{T} \\
& =\mathbf{A}(\omega) \mathbf{y}(\omega) .
\end{aligned}
$$

The matrix $\mathbf{A}(\omega)$, later referred as local filter, is defined as

$$
\mathbf{A}(\omega)=\left[\begin{array}{cccc}
\mathbf{a}^{H}(\omega) & 0 & \ldots & 0 \\
0 & \mathbf{a}^{H}(\omega) & \ldots & 0 \\
\vdots & \vdots & \ddots & \vdots \\
0 & 0 & \ldots & \mathbf{a}^{H}(\omega)
\end{array}\right] .
$$

Notice that in (14) and (15) we assumed that all the FOSDMs are filtered with the same filter, i.e.

$$
\mathbf{a}^{(1)}(\omega)=\mathbf{a}^{(2)}(\omega)=\ldots=\mathbf{a}^{(K)}(\omega)=\mathbf{a}(\omega) .
$$

After combining (9), (12), (14) and (15), we obtain

$$
\begin{aligned}
T(\omega)= & \mathbf{h}^{H}(\omega, \beta) \mathbf{A}(\omega) \mathbf{y}(\omega) \\
= & \mathbf{h}^{H}(\omega, \beta) \mathbf{A}(\omega) \mathbf{d}(\omega, \theta) X(\omega)+ \\
& \mathbf{h}^{H}(\omega, \beta) \mathbf{A}(\omega) \mathbf{v}(\omega) .
\end{aligned}
$$

Notice that in (17) we can easily distinguish the two aforementioned (local and global) filtering stages characterizing this approach. Local filtering is performed by multiplying the vector $\mathbf{y}(\omega)$ by the matrix $\mathbf{A}(\omega)$, while global filtering is performed multiplying the result of local filtering $\mathbf{z}(\omega)=\mathbf{A}(\omega) \mathbf{y}(\omega)$ by the vector $\mathbf{h}^{H}(\omega)$. In Section III we will discuss possible strategies for the design of such filters.

\section{Performance Measures}

In order to design spatial filters, it is useful to define a set of performance measures. For notational convenience we introduce a general spatial filter vector $\mathbf{f}(\omega, \beta)$ that represents a spatial filter designed to steer a beam towards the angle $\beta$, which can be equal to $\mathbf{w}(\omega)$ or to $\mathbf{A}(\omega)^{H} \mathbf{h}(\omega)$, according to the adopted design strategy. Given the steering vector $\mathbf{d}(\omega, \beta)$, we can define the following performance measures [5]:

- White Noise Gain (WNG):

$$
\mathrm{WNG}[\mathbf{f}(\omega, \beta)]=\frac{\left|\mathbf{f}^{H}(\omega, \beta) \mathbf{d}(\omega, \beta)\right|^{2}}{\mathbf{f}^{H}(\omega, \beta) \mathbf{f}(\omega, \beta)},
$$

- Directivity Factor (DF):

$$
\operatorname{DF}[\mathbf{f}(\omega, \beta)]=\frac{\left|\mathbf{f}^{H}(\omega, \beta) \mathbf{d}(\omega, \beta)\right|^{2}}{\mathbf{f}^{H}(\omega, \beta) \boldsymbol{\Gamma}_{\mathrm{dn}}(\omega) \mathbf{f}(\omega, \beta)},
$$

where

$$
\begin{aligned}
{\left[\boldsymbol{\Gamma}_{\mathrm{dn}}(\omega)\right]_{i j} } & =\frac{\sin \left[\omega \rho_{i j} / c\right]}{\omega \rho_{i j} / c} \\
& =\operatorname{sinc}\left[\omega \rho_{i j} / c\right],
\end{aligned}
$$

and $\rho_{i j}$ is the distance between the $i$ th and $j$ th microphones. 
These metrics quantify the gain in SNR (i.e., the ratio between the output SNR and input SNR) achieved by the spatial filters; the WNG assumes a spatially white noise, while the DF assumes a diffuse noise.

\section{Design of the Spatial Filters}

In this section we first derive an expression for the general spatial filter $\mathbf{f}(\omega, \beta)$ and then we specialize its design for the two filtering approaches discussed in Subsection III-B

A spatial filter that aims at minimizing the noise while keeping the signal coming from a given direction $\beta$ unchanged is obtained by solving the following optimization problem [25. p. 279]

$$
\begin{array}{cl}
\underset{\mathbf{f}(\omega, \beta)}{\arg \min } & \mathbf{f}^{H}(\omega, \beta) \mathbf{R}(\omega) \mathbf{f}(\omega, \beta) \\
\text { subject to } & \mathbf{f}^{H}(\omega, \beta) \mathbf{d}(\omega, \beta)=1,
\end{array}
$$

where $\mathbf{R}(\omega)=E\left[\mathbf{v}(\omega) \mathbf{v}^{H}(\omega)\right]$ is the noise covariance matrix and $E[\cdot]$ the expectation operator. The solution to 21$]$ is given by [25, p. 354]

$$
\mathbf{f}(\omega, \beta)=\frac{\mathbf{R}^{-1}(\omega) \mathbf{d}(\omega, \beta)}{\mathbf{d}^{H}(\omega, \beta) \mathbf{R}^{-1}(\omega) \mathbf{d}(\omega, \beta)} .
$$

\section{A. Direct Filtering Approach}

In order to design the filter $\mathbf{w}(\omega, \beta)$ in (10), where the dependence on the steering angle $\beta$ has been made explicit, we can simply set $\mathbf{f}(\omega, \beta)=\mathbf{w}(\omega, \beta)$ in (21) and (22). If we assume that the noise at the microphones is spatially white, we have that $\mathbf{R}(\omega)=\mathbf{I}$, with $\mathbf{I}$ the identity matrix. This is the assumption behind a standard DAS beamformer, which leads to the spatial filter [25] p. 277]

$$
\mathbf{w}(\omega, \beta)_{\text {DAS }}=\frac{\mathbf{d}(\omega, \beta)}{\mathbf{d}^{H}(\omega, \beta) \mathbf{d}(\omega, \beta)} .
$$

Filter (23) maximizes the WNG. Instead, if we assume that the noise at the microphones is diffuse (i.e., $\mathbf{R}(\omega)=\boldsymbol{\Gamma}_{\mathrm{dn}}(\omega)$ ), we obtain a SD spatial filter as [25, p. 280]

$$
\mathbf{w}(\omega, \beta)_{\mathrm{SD}}=\frac{\boldsymbol{\Gamma}_{\mathrm{dn}}^{-1}(\omega) \mathbf{d}(\omega, \beta)}{\mathbf{d}^{H}(\omega, \beta) \boldsymbol{\Gamma}_{\mathrm{dn}}^{-1}(\omega) \mathbf{d}(\omega, \beta)},
$$

which maximizes the DF. As underlined in [26]-[28] the design in 24) is sensitive to the spatially white noise, hence a regularization term is usually added in the design as follows

$$
\mathbf{w}(\omega, \beta)_{\mathrm{SD}}=\frac{\left[\boldsymbol{\Gamma}_{\mathrm{dn}}(\omega)+\epsilon \mathbf{I}\right]^{-1} \mathbf{d}(\omega, \beta)}{\mathbf{d}^{H}(\omega, \beta)\left[\boldsymbol{\Gamma}_{\mathrm{dn}}(\omega)+\epsilon \mathbf{I}\right]^{-1} \mathbf{d}(\omega, \beta)},
$$

where $\epsilon \geq 0$ is the regularization parameter and $\mathbf{I}$ the identity matrix. In the literature, the regularization term in 25) has been used to progressively change the behavior of the spatial filter from a DAS to a SD beamformer (e.g., [28]). Such an approach requires a matrix inversion in order to compute the filter coefficients. In the following sections, we will show that a similar result can be achieved using an alternative approach which does not require any matrix inversion and does employ a single parameter related to the directivity of the local FOSDMs.
It is worth noticing that the definitions of spatial filters in this subsection are all special cases of (22); however, their explicit derivation will reveal useful, since we will refer to each one of them in the next sections.

\section{B. Two-Stage Filtering Approach}

1) First stage (local filter design): The first stage of the two-stage approach concerns the filtering of the FOSDM and hence the design of the filter $\mathbf{a}(\omega)$ in (5). As shown in [23], [29] the vector $\mathbf{a}(\omega)$ can be conveniently expressed in the form

$$
\mathbf{a}(\omega, p, \phi)=p \mathbf{q}_{o}+(1-p) \mathbf{q}_{d}(\omega, \phi),
$$

where $p \in[0,1]$ is a scalar parameter that controls the shape of the resulting beampattern, $\mathbf{q}_{o}$ and $\mathbf{q}_{d}$ represent the normalized response of a monopole and a dipole oriented towards $\phi \in$ $[0,2 \pi)$, respectively. These responses are given by

$$
\begin{aligned}
\mathbf{q}_{o} & =[1 / 4,1 / 4,1 / 4,1 / 4]^{T} \\
\mathbf{q}_{d}(\omega, \phi) & =\frac{c}{j 2 \omega r}\left(\cos (\phi)\left[\begin{array}{c}
1 \\
0 \\
-1 \\
0
\end{array}\right]+\sin (\phi)\left[\begin{array}{c}
0 \\
1 \\
0 \\
-1
\end{array}\right]\right) .
\end{aligned}
$$

Such a design is very flexible since it allows us to steer the beam in any direction and with an arbitrary first-order beampattern by simply modifying the value of $\phi$ and $p$, respectively. Moreover, expressing filter $\mathbf{a}(\omega, p, \phi)$ in the form 26) will become handy in Section III-C, where we find the conditions to decouple local and global filters.

Given the definition of $\mathbf{a}(\omega, p, \phi)$ in 26 the definition of $\mathbf{A}(\omega)$ in $(15)$ can be rewritten as

$$
\begin{aligned}
\mathbf{A}= & p\left[\begin{array}{cccc}
\mathbf{q}_{o}^{H} & 0 & \ldots & 0 \\
0 & \mathbf{q}_{o}^{H} & \ldots & 0 \\
\vdots & \vdots & \ddots & \vdots \\
0 & 0 & \ldots & \mathbf{q}_{o}^{H}
\end{array}\right]+ \\
& (1-p)\left[\begin{array}{cccc}
\mathbf{q}_{d}^{H} & 0 & \ldots & 0 \\
0 & \mathbf{q}_{d}^{H} & \ldots & 0 \\
\vdots & \vdots & \ddots & \vdots \\
0 & 0 & \ldots & \mathbf{q}_{d}^{H}
\end{array}\right]= \\
= & p \mathbf{Q}_{o}+(1-p) \mathbf{Q}_{d},
\end{aligned}
$$

where the dependency on the variable $\omega, p$ and $\phi$ is here omitted for the sake of readability.

2) Second stage (global filter design): The second stage concerns the design of the filter $\mathbf{h}(\omega, \beta)$ in (17). For the design of this filter we can follow an approach similar to the one adopted in the direct filtering approach. In particular, we assume that the noise covariance matrix of the uniform linear array of FOSDMs is an identity matrix. Hence, in order to find the coefficients of the global filter, we solve the following optimization problem

$$
\begin{array}{ll}
\underset{\mathbf{h}(\omega, \beta)}{\arg \min } & \mathbf{h}^{H}(\omega, \beta) \mathbf{h}(\omega, \beta) \\
\text { subject to } & \mathbf{h}^{H}(\omega, \beta) \mathbf{A}(\omega, p, \phi) \mathbf{d}(\omega, \beta)=1,
\end{array}
$$


whose solution is [25] p. 354]

$$
\mathbf{h}(\omega, \beta)=\frac{\mathbf{A}(\omega, p, \phi) \mathbf{d}(\omega, \beta)}{\mathbf{d}^{H}(\omega, \beta) \mathbf{A}^{H}(\omega, p, \phi) \mathbf{A}(\omega, p, \phi) \mathbf{d}(\omega, \beta)}
$$

It is worth noting that the steering angle $\beta$ of the filter $\mathbf{h}(\omega)$ is in principle different to the steering angle $\phi$ of the FOSDMs.

\section{Decoupling of Local Filter and Global Filter}

From (30), we notice that the global filter $\mathbf{h}(\omega, \beta)$ is a function of the local filter $\mathbf{A}(\omega, p, \phi)$.

For reasons of computational efficiency and design flexibility, it would be desirable to have expressions of the global filter and of the local filter which are independent one another. In this subsection we show that, under certain conditions, the two filters $\mathbf{h}(\omega, \beta)$ and $\mathbf{A}(\omega, p, \phi)$ can indeed be decoupled. Let us consider the ideal case in which the global filter can be designed as a DAS beamformer with $K$ sensors arranged in an uniform linear array with inter-element spacing $\delta$. In this case, the global filter would be defined as

$$
\mathbf{h}(\omega, \beta)=\frac{\mathbf{g}(\omega, \beta)}{\mathbf{g}^{H}(\omega, \beta) \mathbf{g}(\omega, \beta)},
$$

where $\mathbf{g}(\omega, \beta)$ is the steering vector of the uniform linear array, defined as

$$
\begin{array}{r}
\mathbf{g}(\omega, \beta)=\left[e^{-j \frac{\omega}{c} \sin (\beta) \delta\left(-\frac{(K-1)}{2}\right)}, e^{-j \frac{\omega}{c} \sin (\beta) \delta\left(1-\frac{(K-1)}{2}\right),}\right. \\
\left.\ldots, e^{-j \frac{\omega}{c} \sin (\beta) \delta\left(\frac{(K-1)}{2}\right)}\right]^{T} .
\end{array}
$$

Clearly, 31 does not depend on $\mathbf{A}(\omega, p, \phi)$. Let us now investigate under which conditions we can have (30) matching (31), which corresponds to studying the conditions of validity of

$$
\mathbf{A}(\omega, p, \phi) \mathbf{d}(\omega, \beta)=\mathbf{g}(\omega, \beta) .
$$

As proven in Appendix A, (33) is valid if $\lambda=c / f \gg r$ and $\phi=\beta$. The condition $\lambda \gg r$ is met when the radius $r$ of FOSDMs is small with respect to the wavelength $\lambda$. This is assumed to be valid with FOSDMs for the full bandwidth of the signal of interest. The condition $\phi=\beta$, instead, requires that the steering angle of the global filter $\beta$ and the one of the local filter $\phi$ match. Notice that these two conditions do not constrain the value of the beampattern shape parameter $p$, which, therefore, remains a free parameter. The following section describes possible strategies for the design of $p$.

\section{Design of the FOSDM BeAmpattern Shape PARAMETER}

In this section we discuss two strategies, based on the solution of optimization problems, for designing the FOSDM beampattern shape parameter $p$. The first leads to a value of $p$ that is frequency dependent, whereas in the second the beampattern shape parameter is a frequency-independent coefficient. In all cases the cost function of the optimization problem is defined as the squared error between a filter computed using the direct filtering approach in Subsection III-A and a filter computed using the proposed two-stage approach.
It is useful to make explicit the dependence of the filter $\mathbf{A}^{H}(\omega, p, \phi) \mathbf{h}(\omega, \beta)$ on the beampattern shape parameter $p$ as

$$
\begin{aligned}
\mathbf{A}^{H}(\omega, p, \phi) \mathbf{h}(\omega, \beta)= & \left(p \mathbf{Q}_{o}+(1-p) \mathbf{Q}_{d}(\omega, \phi)\right)^{H} \mathbf{h}(\omega, \beta) \\
= & p\left(\mathbf{Q}_{o}^{H}-\mathbf{Q}_{d}^{H}(\omega, \phi)\right) \mathbf{h}(\omega, \beta) \\
& +\mathbf{Q}_{d}^{H}(\omega, \phi) \mathbf{h}(\omega, \beta) .
\end{aligned}
$$

\section{A. Frequency-dependent Beampattern Shape Parameter}

In order to find a frequency-dependent beampattern shape parameter, we solve an optimization problem of the form

$$
\underset{p(\omega)}{\arg \min }\left\|\mathbf{w}(\omega, \beta)-\mathbf{A}^{H}(\omega, p, \beta) \mathbf{h}(\omega, \beta)\right\|^{2},
$$

where the variable $\mathbf{w}(\omega, \beta)$ can be replaced by either (23) or (25) for the optimization of the WNG or the DF, respectively. It is worth noting that in (35) we set $\beta$ equal to $\phi$ in order to fulfil the condition for the decoupling of the local and global filters discussed in Section [III-C Making explicit the dependence on $p$ using (34), and defining two auxiliary variables

$$
\begin{aligned}
\boldsymbol{\kappa}(\omega) & =\mathbf{w}(\omega, \beta)-\mathbf{Q}_{d}^{H}(\omega, \beta) \mathbf{h}(\omega, \beta) \\
\boldsymbol{\ell}(\omega) & =\left(\mathbf{Q}_{o}^{H}-\mathbf{Q}_{d}^{H}(\omega, \beta)\right) \mathbf{h}(\omega, \beta),
\end{aligned}
$$

we can easily rewrite the optimization problem in 35 in the form

$$
\underset{p(\omega)}{\arg \min }\|\boldsymbol{\kappa}(\omega)-p(\omega) \boldsymbol{\ell}(\omega)\|^{2} .
$$

By solving the optimization problem in (37), we obtain the frequency-dependent beampattern shape parameter $p(\omega)$ as follows [30], [31]

$$
p(\omega)=\operatorname{Re}\left\{\frac{\ell^{H}(\omega) \boldsymbol{\kappa}(\omega)}{\ell^{H}(\omega) \boldsymbol{\ell}(\omega)}\right\},
$$

where the operator $\operatorname{Re}\{\cdot\}$ takes the real part of the argument.

\section{B. Frequency-independent Beampattern Shape Parameter}

The design of a frequency-independent beampattern shape parameter $p$ can be accomplished by following the same rationale of Section IV-A More precisely, if we uniformly sample the frequency axis at $N$ points $\omega_{1}, \ldots, \omega_{N}$, we can find the frequency-independent beampattern shape parameter by solving the optimization problem

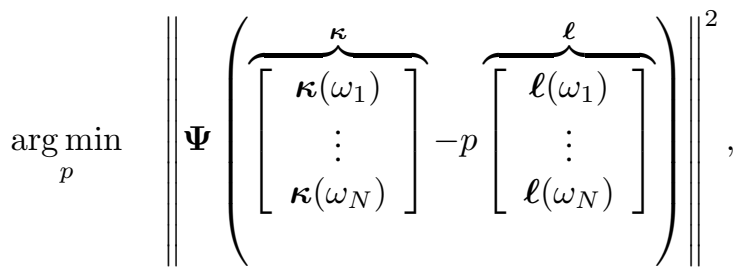

where $\Psi$ is a weighting block diagonal matrix defined as

$$
\Psi=\operatorname{diag}\left(\Psi\left(\omega_{1}\right) \mathbf{I}, \ldots, \Psi\left(\omega_{N}\right) \mathbf{I}\right) .
$$

The solution to this optimization problem is

$$
p=\operatorname{Re}\left\{\frac{\ell^{H} \boldsymbol{\Psi}^{H} \mathbf{\Psi} \boldsymbol{\kappa}}{\ell^{H} \boldsymbol{\Psi}^{H} \boldsymbol{\Psi} \ell}\right\} .
$$


The design of the weights $\Psi\left(\omega_{n}\right)$, with $n=1, \ldots, N$ can be performed in different ways. The most straightforward approach would be to simply set $\Psi\left(\omega_{n}\right)=1, \forall n$. However, the design of the weights proposed in this manuscript is a function of the error obtained in the frequency-dependent case (see (37)). In particular, we set

$$
\begin{aligned}
\Psi\left(\omega_{n}\right) & =\frac{1}{\left\|\boldsymbol{\kappa}\left(\omega_{n}\right)-p\left(\omega_{n}\right) \ell\left(\omega_{n}\right)\right\|^{2}} \\
& =\frac{1}{\left\|\mathbf{w}\left(\omega_{n}, \beta\right)-\mathbf{A}^{H}\left(\omega_{n}, p, \beta\right) \mathbf{h}\left(\omega_{n}, \beta\right)\right\|^{2}},
\end{aligned}
$$

where $p\left(\omega_{n}\right)$ is the result of the optimization problem as given in (38). Weights in (42) are inversely proportional to the squared $L^{2}$ norm of the difference between the optimum filter $\mathbf{A}^{H}\left(\omega_{n}, p, \beta\right) \mathbf{h}\left(\omega_{n}, \beta\right)$ and the target filter $\mathbf{w}\left(\omega_{n}, \beta\right)$.

As in Section IV-A the maximization of WNG and DF is obtained by setting $\mathbf{w}(\omega, \beta)=\mathbf{w}(\omega, \beta)_{\text {DAS }}$ and $\mathbf{w}(\omega, \beta)=$ $\mathbf{w}(\omega, \beta)_{\mathrm{SD}}$, respectively.

\section{ANALYSIS IN TERMS OF WNG AND DF}

In this section we carry out some simulations in order to evaluate the performance of the proposed beamformers. For what concerns the geometry of the array, the parameters are $\delta=8 \mathrm{~cm}, r=1 \mathrm{~cm}$ and $K=16$, respectively. It follows that the total number of omnidirectional sensors is $4 K=64$.

We compare the proposed two-stage filtering approach based on FOSDMs with the direct filtering approach described in Section III-A in terms of WNG and DF. In particular, we analyse the following spatial filters:

- a DAS designed using the approach in (23);

- a filter designed using a weighted frequency-independent beampattern shape parameter (see (39)), where we set $\mathbf{w}(\omega, \beta)=\mathbf{w}(\omega, \beta)_{\text {DAS }}$ in 36, later referred to as "TwoStage-DAS";

- a filter designed using a frequency-dependent beampattern shape parameter (see (37)), where we set $\mathbf{w}(\omega, \beta)=$ $\mathbf{w}(\omega, \beta)_{\text {DAS }}$ in (36), later referred to as "Two-Stage$\mathrm{DAS}_{f}$;

- a SD designed using the approach in 25);

- a filter designed using a weighted frequency-independent beampattern shape parameter (see (39)), where we set $\mathbf{w}(\omega, \beta)=\mathbf{w}(\omega, \beta)_{\mathrm{SD}}$ in $(36$, later referred to as "TwoStage-SD";

- a filter designed using a frequency-dependent beampattern shape parameter (see (37)), where we set $\mathbf{w}(\omega, \beta)=$ $\mathbf{w}(\omega, \beta)_{\mathrm{SD}}$ in (36), later referred to as "Two-Stage-SD ${ }_{f}$ ".

All these filters are designed to steer a beam in the positive direction of the $x$ axis. This corresponds to setting $\beta=0^{\circ}$ in (23), (24) and (36). Moreover, for the filters SD, TwoStage-SD and Two-Stage-SD ${ }_{f}$ the design of $\mathbf{w}(\omega, \beta)_{\mathrm{SD}}$ has been done using 25] with $\epsilon=0.01$. Fig. 3 shows the beampattern shape parameters used in the two-stage filtering approaches under comparison. In particular, Fig. 3a. shows both the frequency-dependent beampattern shape parameter used in the Two-Stage-DAS ${ }_{f}$ and the frequency-independent one used in the Two-Stage-DAS. According to [26, when $p=1$ the FOSDMs behave like omnidirectional sensors. Fig. 3b instead,
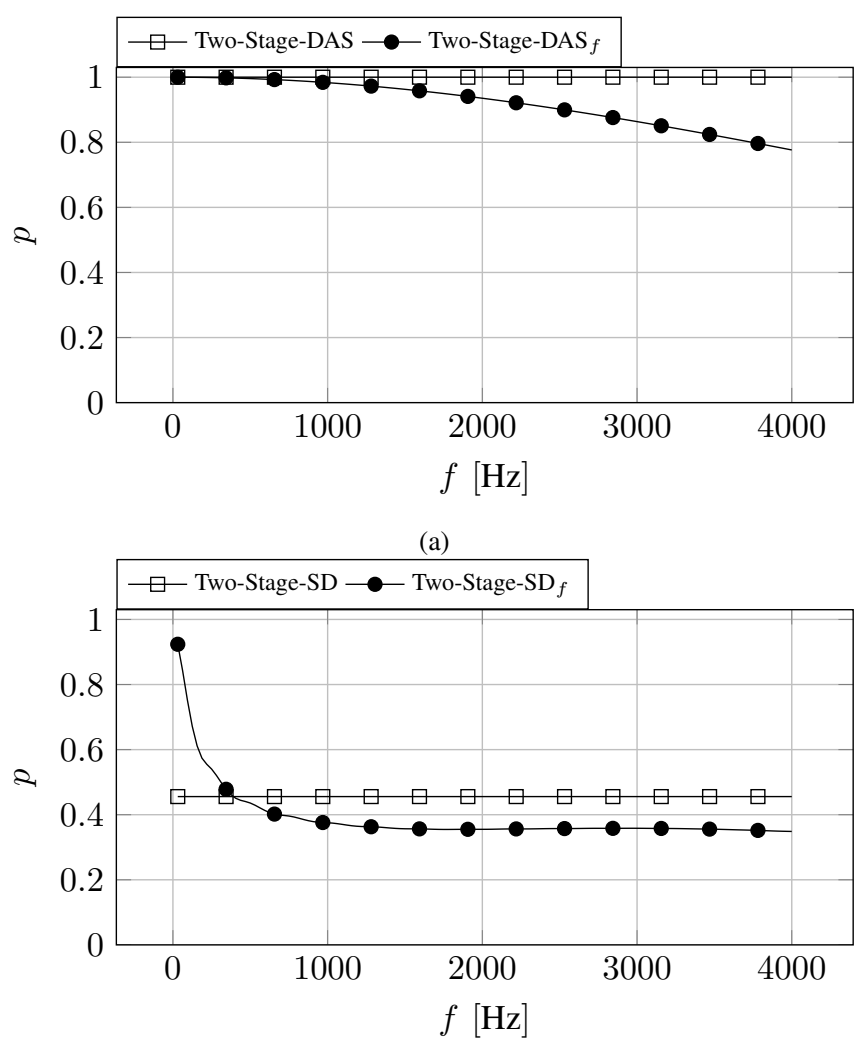

(b)

Fig. 3: Beampattern shape parameter used in the two-stage filtering approach. Fig. 3a shows the Two-Stage-DAS and the Two-Stage$\operatorname{DAS}_{f}$. The Two-Stage-DAS uses a frequency-independent beampattern shape parameter while the Two-Stage-DAS $S_{f}$ a frequencydependent one. Fig. $3 b$ shows the Two-stage-SD and the Two-stage$\mathrm{SD}_{f}$. The Two-Stage-SD uses a frequency-independent beampattern shape parameter while the Two-Stage-SD $\mathrm{S}_{f}$ a frequency-dependent one.

shows the frequency-dependent beampattern shape parameter used in the Two-Stage-SD $\mathrm{SD}_{f}$ and the frequency-independent one used in the Two-Stage-SD.

\section{A. Comparison in terms of $W N G$}

We start with an analysis of the filters in terms of WNG, which, we recall, is maximized by the DAS. The results of the simulations are portrayed in Fig. 4 In particular, Fig. 4a shows a comparison between the WNG of the DAS and two filters obtained with the proposed two-stage filtering approach (TwoStage-DAS and Two-Stage-DAS ${ }_{f}$ ). In Fig. $4 b$ instead, the SD is compared with the Two-Stage-SD and the Two-Stage-SD $f$ in terms of WNG.

As far as Fig. 4a is concerned, we notice that the WNG of the Two-Stage-DAS ${ }_{f}$ closely matches the WNG of the DAS. Even if the WNG of the Two-Stage-DAS is slightly lower at high frequencies, the difference in WNG between the TwoStage-DAS and the Two-Stage-DAS ${ }_{f}$ is remarkably minimal. In Fig. $4 b$, instead, we can see that the WNG of the two-stage filters generally matches or surpasses that of the SD, up to an improvement of WNG of more than $5 \mathrm{~dB}$ at high frequencies. It is worth stressing the fact that, also in this case, the Two- 


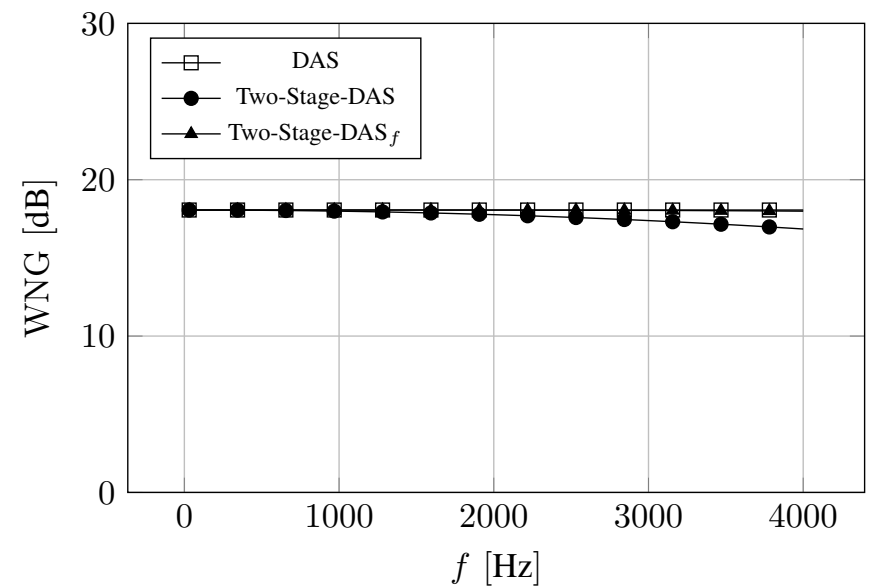

(a)

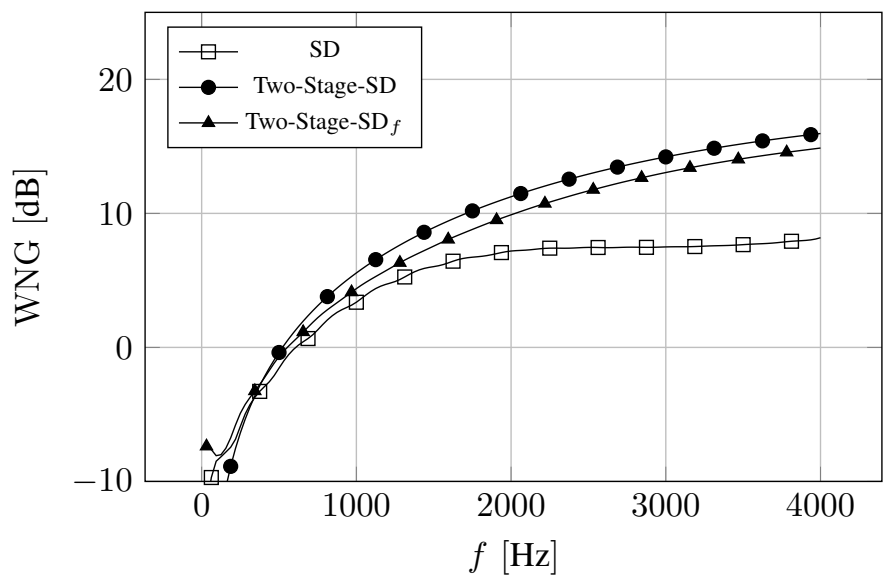

(b)

Fig. 4: Comparison in terms of WNG. In Fig. 4a the DAS is compared with the Two-Stage-DAS and the Two-Stage-DAS $f$. The Two-StageDAS uses a frequency-independent beampattern shape parameter while the Two-Stage-DAS ${ }_{f}$ a frequency-dependent one. In Fig. 4b the SD

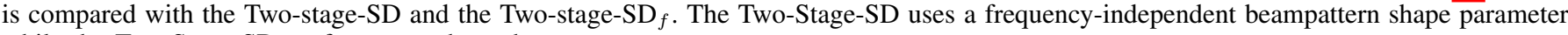
while the Two-Stage-SD $f$ a frequency-dependent one.

Stage-DAS and the Two-Stage-DAS $f$ have a similar trend in the considered frequency range.

\section{B. Comparison in terms of $D F$}

The second comparison concerns the analysis of the performance of the filters in terms of DF, which is maximized by the SD beamformer. Results of the simulations are reported in Fig. 5 .

More precisely, Fig. 5a shows that the Two-Stage-DAS $f$ achieves practically the same performance of the DAS. The Two-stage-DAS, instead, exhibits a slight decrease in DF at higher frequencies with respect to the DAS case. As far as Fig. $5 \mathrm{~b}$ is concerned, we can see that the behavior of the twostage filters closely matches that of the SD in the considered frequency range. Moreover, except at very low frequencies, no significant difference in terms of DF is present between the Two-Stage-SD $f$ and the Two-Stage-SD. To conclude, not only do the proposed two filters behave very similarly to the SD in terms of DF, but they also outperform the SD in terms of WNG, as shown in Fig. $4 b$

\section{Further Metrics AND Results}

In this section we evaluate the proposed spatial filters using metrics that are rather different from WNG and DF. In particular, we perform an analysis in terms of beampattern, Frontto-Back Ratio and two newly defined metrics that evaluate the dependence of DF on frequency and steering angle.

\section{A. Beampattern}

The beampattern of a beamformer characterized by a spatial filter $\mathbf{f}(\omega, \beta)$ is defined as

$$
\mathcal{B}[\mathbf{f}(\omega, \beta), \theta]=\mathbf{f}^{H}(\omega, \beta) \mathbf{d}(\omega, \theta) .
$$

In Fig. 6 and Fig. 7 we compare beampatterns obtained using the proposed two-stage filtering approach and the direct filtering approach. Beampatterns are evaluated at different frequencies and steering angles. In particular, Fig. 6 shows the comparison between the beampatterns of a DAS, a Two-StageDAS and a Two-Stage-DAS $f$, which are nearly symmetric with respect to the $y$ axis. It is worth noticing that the beampatterns of the two-stage filters closely match the one of the DAS. Fig. 77 instead, shows the comparison between the beampatterns of a SD, a Two-Stage-SD and a Two-Stage$\mathrm{SD}_{f}$, which are non-symmetric and highly directive. Also in this case the beampatterns of the two-stage filters closely match the one of the SD. Mismatches in terms of sidelobe level slightly increase as the steering angle deviates from the broadside direction.

\section{B. Front-to-Back Ratio}

The Front-to-Back Ratio (FBR) measures the ability of a spatial filter to attenuate signals coming from the back of the array with respect to the frontal direction [5]. The "front" and the "back" are defined with respect to a reference steering angle $\beta$. Formally, we express the FBR as

$$
\operatorname{FBR}[\mathbf{f}(\omega, \beta)]=\frac{\int_{\beta-\pi / 2}^{\beta+\pi / 2}|\mathcal{B}[\mathbf{f}(\omega, \beta), \theta]|^{2} d \theta}{\int_{\beta+\pi / 2}^{\beta+3 \pi / 2}|\mathcal{B}[\mathbf{f}(\omega, \beta), \theta]|^{2} d \theta},
$$

where $\mathcal{B}[\mathbf{f}(\omega, \beta), \theta]$ is the beampattern defined in (43). Results are shown in Fig. 8 for the same set of filters defined in Section $\mathrm{V}$. In accordance to Section $\mathrm{V}, \beta=0^{\circ}$.

In particular, Fig. 8a shows that the DAS and the TwoStage-DAS $f$ exhibit an almost identical trend. On the other hand, the FBR of the Two-Stage-DAS yields $0 \mathrm{~dB}$ for all frequencies. This can be explained by the fact that, in this case, the beampattern shape parameter is such that all the FOSDMs are characterized by an omnidirectional directivity pattern, i.e., $p=1$, (see Fig. 3a). As a consequence our system can be thought of as an uniform linear array of $K$ omnidirectional sensors that presents a symmetrical beampattern (i.e., $0 \mathrm{~dB}$ FBR at all the frequencies). 


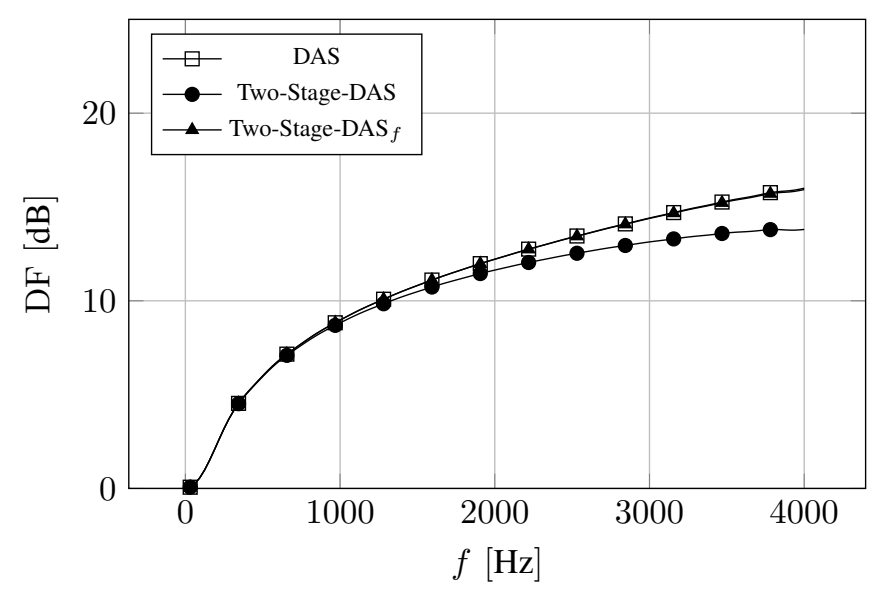

(a)

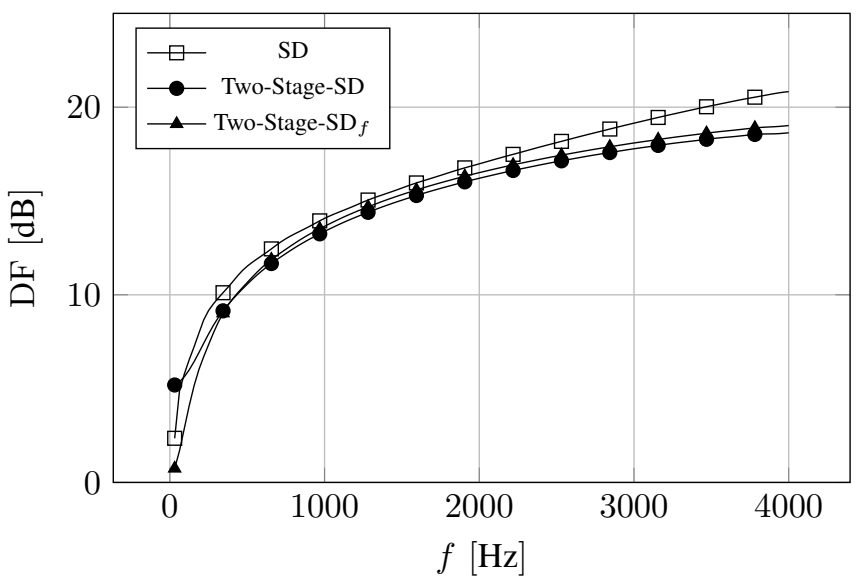

(b)

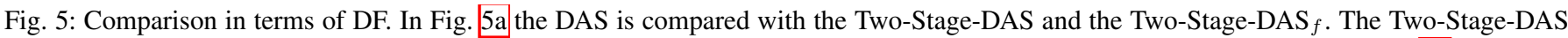
uses a frequency-independent beampattern shape parameter while the Two-Stage-DAS ${ }_{f}$ a frequency-dependent one. In Fig. 5b the SD is

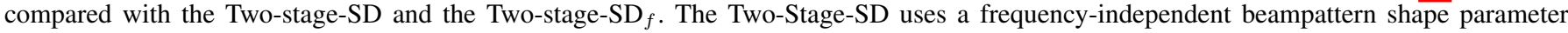
while the Two-Stage-SD $f$ a frequency-dependent one.

As expected, Fig. $8 \mathrm{~b}$ shows that, with respect to the results in Fig. 8a, the FBR increases for all the analyzed filters. In particular, the Two-Stage-SD outperforms the others with a gap of almost $10 \mathrm{~dB}$ below $2 \mathrm{kHz}$.

\section{Directivity Factor Frequency Variance}

Let us now define a metric that computes the variability of the DF along the frequency axis for different steering angles and call it Directivity Factor Frequency Variance (DFFV). The purpose of this metric is to evaluate "how frequency-invariant the filter is". Formally, the DFFV is defines as

$$
\operatorname{DFFV}[\mathbf{f}(\beta)]=\frac{1}{N-1} \sum_{n=1}^{N}\left|\operatorname{DF}\left[\mathbf{f}\left(\omega_{n}, \beta\right)\right]-\mu(\beta)\right|^{2},
$$

with $\mu(\beta)=1 / N \sum_{n=1}^{N} \operatorname{DF}\left[\mathbf{f}\left(\omega_{n}, \beta\right)\right]$. Results are shown in Fig. 9 for the set of filters defined in Section $\nabla$. We vary the steering angle of the filters in a range from $0^{\circ}$ to $180^{\circ}$.

From the results reported in Fig. 9a, we see that the DAS and the Two-Stage-DAS ${ }_{f}$ are characterized by nearly the same DFFV. The Two-Stage-DAS, instead, has a lower DFFV for all the steering angles.

Fig. 9b shows that the two-stage filters outperform the SD. Moreover, the Two-Stage-SD achieves better results for all the considered steering angles.

\section{Directivity Factor Angle Variance}

We define a further metric which computes the variance of the DF for all the steering angles as a function of frequency and we call it Directivity Factor Angle Variance (DFAV). Similarly to the DFFV, the purpose of this metric is to evaluate "how invariant with respect to the steering angle a filter is". The DFAV is defined as

$$
\operatorname{DFAV}[\mathbf{f}(\omega)]=\frac{1}{I-1} \sum_{i=1}^{I}\left|\operatorname{DF}\left[\mathbf{f}\left(\omega, \beta_{i}\right)\right]-\mu(\omega)\right|^{2},
$$

where $\beta_{i}=(i-1) \pi / I$ and $\mu(\omega)=1 / I \sum_{i=1}^{I} \operatorname{DF}\left[\mathbf{f}\left(\omega, \beta_{i}\right)\right]$. Results are shown in Fig. 10 for the set of filters defined in Section $\nabla$

Fig. 10a shows that the three filters are characterized by nearly the same DFAV in the considered frequency range.

On the other hand, in Fig. $10 b$ we can see that the DFAV of the Two-Stage-SD $f$ and of the SD exhibit a similar trend. The Two-Stage-SD, instead, outperforms the other two for all the frequency bands with a DFAV gap up to almost $10 \mathrm{~dB}$.

\section{Computational Schemes of the Proposed SPATIAL FILTERS}

In this section we describe two possible computational schemes of the proposed spatial filters. We start with the most straightforward implementation that strictly follows the formulation described in the previous sections. A block diagram of this implementation is shown in Fig. 11] As we can see, the computation of the local filter is separated from the one of the global filter. In particular, on the left-hand side of the $k$ th FOSDM (represented by four circles indicating the omnidirectional sensors), two multipliers are used to compute the response of a monopole weighted by $p$. The other operators on the right-hand side, instead, are used to compute the dipole steered toward $\beta$ and weighted by $(1-p)$. The weighted monopole and the weighted dipole are summed to give the FOSDM output. Finally, the output of the $k$ th FOSDM is multiplied by the coefficient $H_{k}^{*}(\omega, \beta)$ of the global filter, and then summed to the other FOSDM signals to give the output of the system.

However, the implementation in Fig. 11 is not unique since many other equivalent implementations can be derived. Indeed, since all the involved operators are linear, we can swap the order of the blocks without affecting the final result.

It follows that one can search for the implementation structure that best fulfills the requirements of the reference application scenario. 


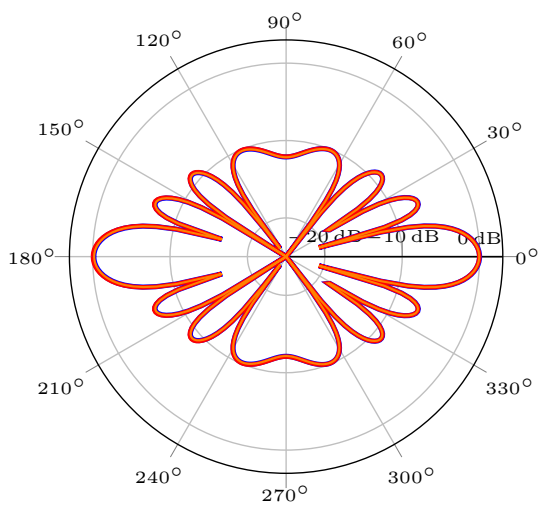

(a) $1 \mathrm{kHz}$

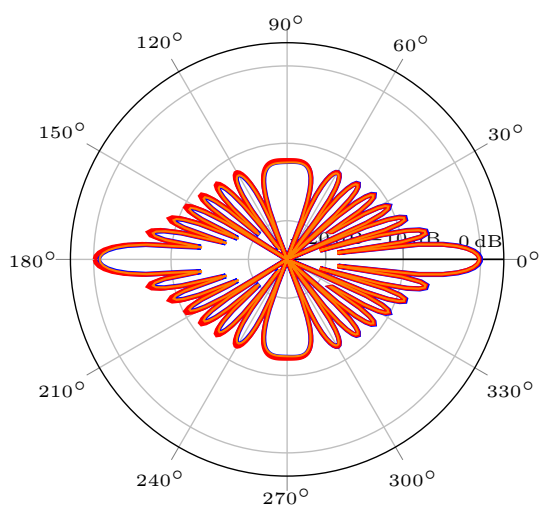

(d) $2 \mathrm{kHz}$

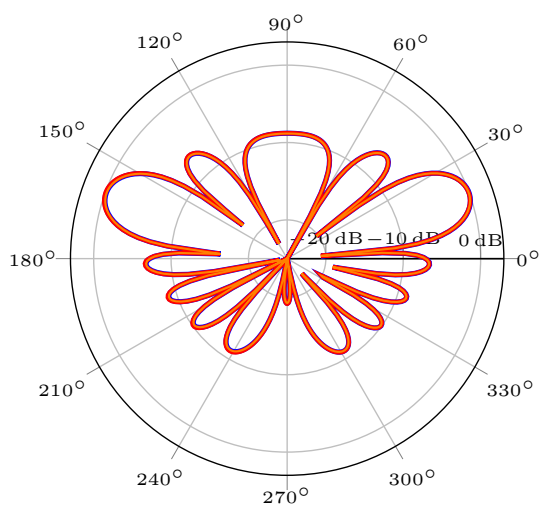

(b) $1 \mathrm{kHz}$

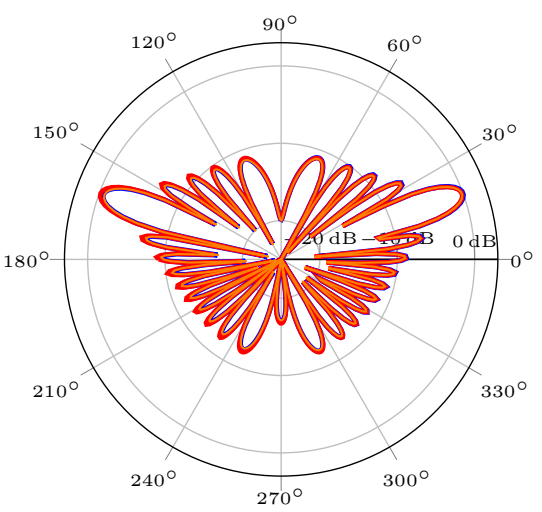

(e) $2 \mathrm{kHz}$

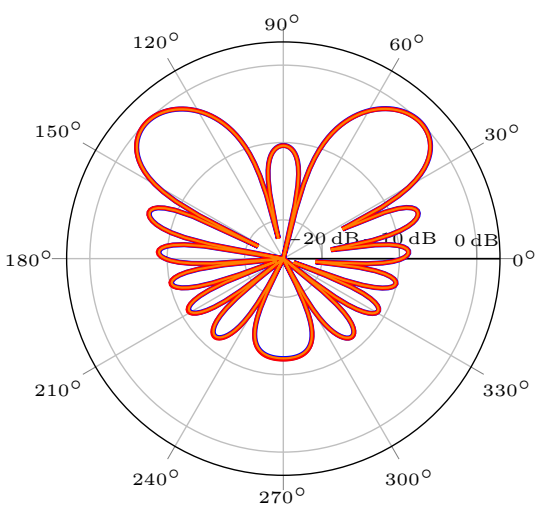

(c) $1 \mathrm{kHz}$

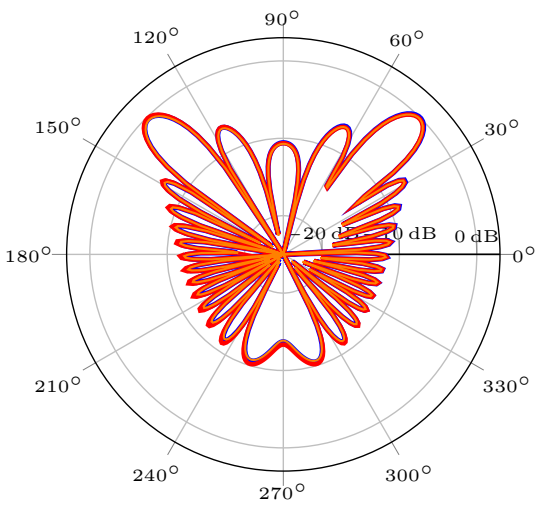

(f) $2 \mathrm{kHz}$

Fig. 6: Polar plots in $\mathrm{dB}$ scale of the beampatterns steered toward $0, \pi / 9$ and $\pi / 4$ at 1 and $2 \mathrm{kHz}$. Each plot contains three beampattern

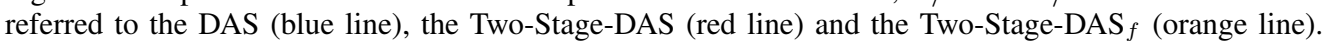

As an example, we derive the block diagram in Fig. 12 which provides the same output of that in Fig. 11] It is worth noticing that, when the beampattern shape parameter is frequency-independent, the implementation scheme in Fig. 12 allows us to perform the "morphing" from a DAS-like beamformer to a SD-like beamformer in an efficient fashion. In fact, the morphing just requires to update the value of $p$ and to perform two operations (a multiplication and an addition).

\section{CONCLUSIONS AND Future WORKS}

In this article we investigated a beamforming technique for linear arrays of First-Order Differential Microphones. We proposed a two-stage filtering approach and we analysed its performance by comparing it with traditional beamforming techniques. We proved that, under certain conditions, the two stages of the proposed filtering approach can be decoupled. The resulting filter is a flexible beamformer that, with a suitable implementation scheme, can efficiently morph between a Delay-and-Sum-like beamformer and a Super-Directivelike beamformer in a continuous fashion. Comparisons with standard beamforming techniques returned promising results. In particular, unlike the standard Super-Directive beamformer, our filter does not involve any matrix inversion that can cause problems due to ill-conditioning. Moreover, as our approach considers the system as an array of FOSDMs, it generally has a lower frequency and steering angle variance of the
Directivity Factor with respect to the Delay-and-Sum and the Super-Directive beamformer.

It is worth saying that, when the inter-element spacing between adjacent FOSDMs $\delta$ is reduced, the DF of the proposed beamformer decreases with respect to a standard SD, while the WNG increases. However, reducing $\delta$ up to the case in which it becomes comparable with the FOSDM radius is generally of little interest. In this regard, note that, reducing the inter-element spacing while keeping fixed the number of FOSDMs, decreases the length of the array, hence its spatial resolution.

As far as future developments are concerned, we are planning to investigate how the proposed approach can be generalized to geometries that differ from uniform linear array and to higher order steerable differential microphones.

\section{APPENDIX A \\ DECOUPLING BETWEEN FOSDM AND GLOBAL FILTERS}

In this Appendix we want to determine the conditions under which we can write that

$$
\mathbf{A}(\omega, p, \phi) \mathbf{d}(\omega, \beta)=\mathbf{g}(\omega, \beta) .
$$

Since this equality involves two vectors, it is verified if and only if the vector elements are pair-wise equal. However, looking at the definition of $\mathbf{A}(\omega, p, \phi), \mathbf{d}(\omega, \beta)$ and $\mathbf{g}(\omega, \beta)$, we can see that all the elements of the vectors $\mathbf{A}(\omega, p, \phi) \mathbf{d}(\omega, \beta)$ 


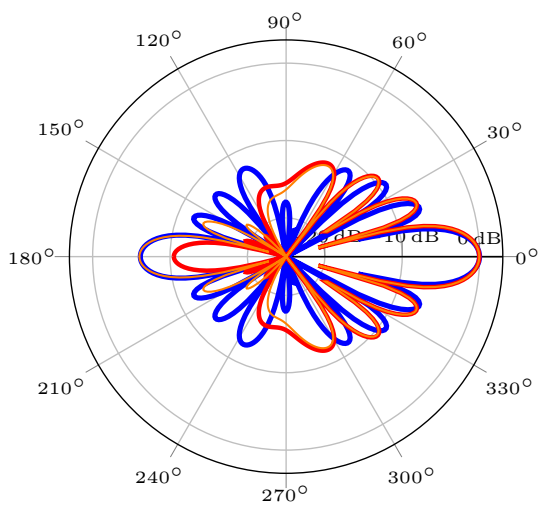

(a) $1 \mathrm{kHz}$

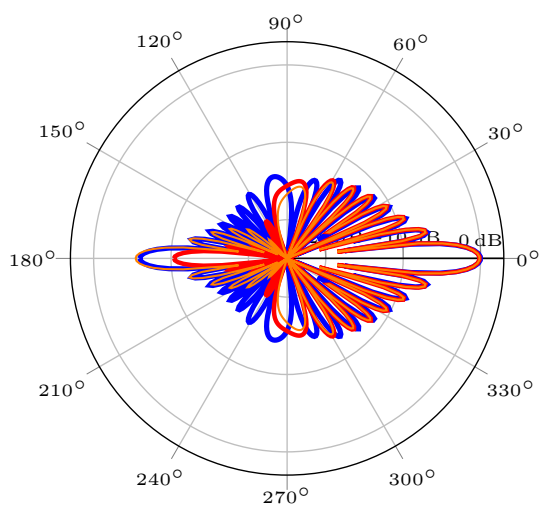

(d) $2 \mathrm{kHz}$

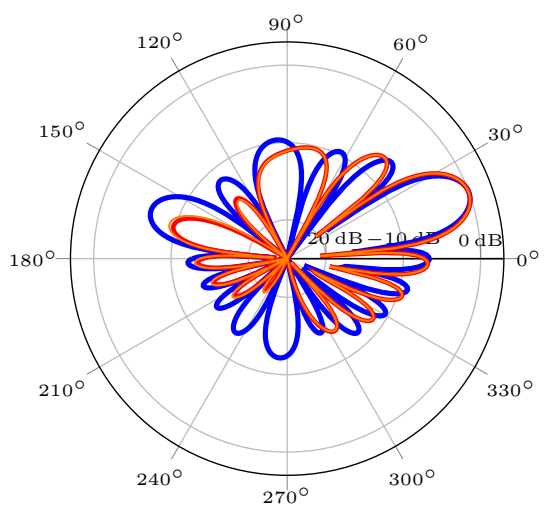

(b) $1 \mathrm{~Hz}$

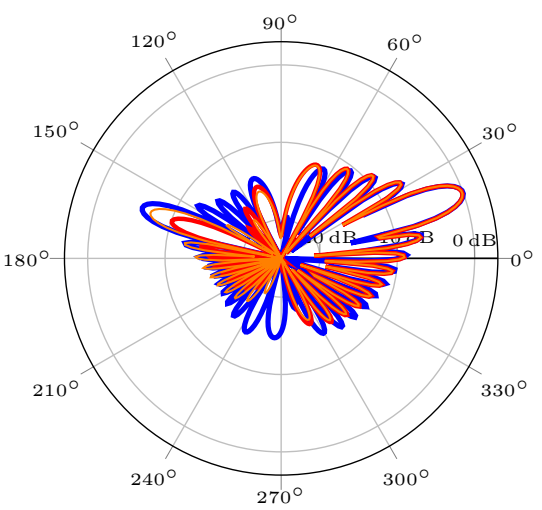

(e) $2 \mathrm{~Hz}$

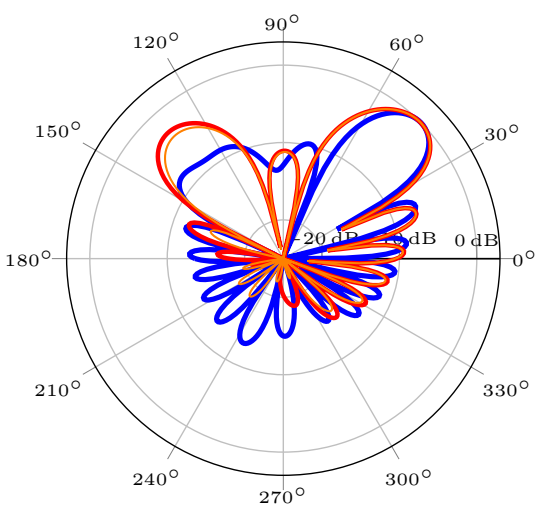

(c) $1 \mathrm{~Hz}$

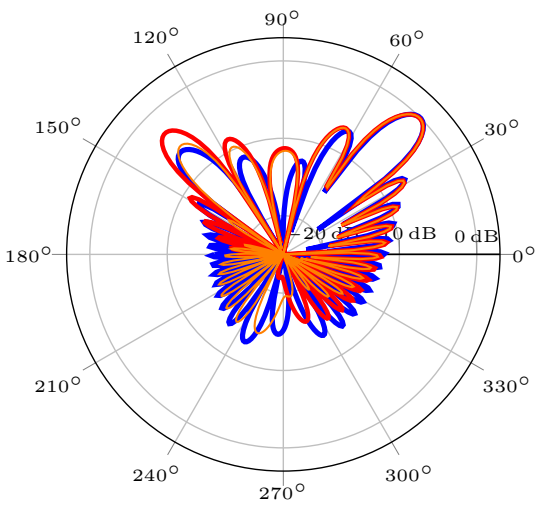

(f) $2 \mathrm{~Hz}$

Fig. 7: Polar plot in $\mathrm{dB}$ scale of the beampatterns steered toward $0, \pi / 9$ and $\pi / 4$ at 1 and $2 \mathrm{kHz}$. Each plot contains three beampattern referred to the SD (blue line), the Two-Stage-SD (red line) and the Two-Stage-SD $($ orange line).

and $\mathbf{g}(\omega, \beta)$ have the same expression except for the FOSDM index $k$. Hence, we can limit ourselves to verify that the equality holds for one arbitrary element of the array. In particular, for reasons of convenience in the derivation and without loss of generality, we choose $k=\frac{K-1}{2}$, for which the corresponding element in the vector $\mathbf{g}(\omega, \beta)$ (see (32) is equal to one (i.e. $[\mathbf{g}(\omega, \beta)]_{k=\frac{K-1}{2}}=1$ ). The corresponding element of the vector $\mathbf{A}(\omega, p, \phi)^{2} \mathbf{d}(\omega, \beta)$ is given by

$$
\begin{aligned}
& \left(p \mathbf{q}_{o}^{T}+(1-p) \mathbf{q}_{d}^{H}(\omega, \phi)\right) \times \\
& {\left[e^{-j \frac{\omega}{c}[\cos (\beta) r]}, e^{-j \frac{\omega}{c}[\sin (\beta) r]}, e^{j \frac{\omega}{c}[\cos (\beta) r]}, e^{j \frac{\omega}{c}[\sin (\beta) r]}\right]=} \\
= & \frac{p}{4}\left(e^{-j \frac{\omega}{c}[\cos (\beta) r]}+e^{-j \frac{\omega}{c}[\sin (\beta) r]}+e^{j \frac{\omega}{c}[\cos (\beta) r]}+e^{j \frac{\omega}{c}[\sin (\beta) r]}\right)+ \\
& -\frac{(1-p) c}{j 2 \omega r}\left(\cos (\phi)\left(e^{-j \frac{\omega}{c}[\cos (\beta) r]}-e^{j \frac{\omega}{c}[\cos (\beta) r]}\right)+\right. \\
& \left.+\sin (\phi)\left(e^{-j \frac{\omega}{c}[\sin (\beta) r]}-e^{j \frac{\omega}{c}[\sin (\beta) r]}\right)\right) .
\end{aligned}
$$

Using the Euler's formula, (48) simplifies to

$$
\begin{aligned}
& \frac{p}{2}\left[\cos \left(\frac{\omega r}{c} \cos (\beta)\right)+\cos \left(\frac{\omega r}{c} \sin (\beta)\right)\right] \\
& +\frac{(1-p) c}{\omega r}\left[\cos (\phi) \sin \left(\frac{\omega r}{c} \cos (\beta)\right)+\right. \\
& \left.+\sin (\phi) \sin \left(\frac{\omega r}{c} \sin (\beta)\right)\right] .
\end{aligned}
$$

Since we are dealing with FOSDMs, we can make the follow- ing assumption, which is typical in the design of DM [5]

$$
\lambda=\frac{2 \pi c}{\omega} \gg r \Rightarrow \frac{\omega r}{c} \rightarrow 0 .
$$

Under this assumption and using the small-angle approximations

$$
\begin{aligned}
& \sin (x) \approx x \\
& \cos (x) \approx 1,
\end{aligned}
$$

the expression in (49) reduces to

$$
\begin{aligned}
& \frac{p}{2}(1+1)+\frac{(1-p) c}{\omega r}\left(\cos (\phi)\left(\frac{\omega}{c}[\cos (\beta) r]\right)+\right. \\
& \left.\sin (\phi)\left(\frac{\omega}{c}[\sin (\beta) r]\right)\right)= \\
& p+(1-p)(\cos (\phi) \cos (\beta)+\sin (\phi) \sin (\beta))= \\
& p+(1-p) \cos (\phi-\beta) .
\end{aligned}
$$

The condition

$$
p+(1-p) \cos (\phi-\beta)=1,
$$

is verified for

$$
\beta=\phi,
$$

which demonstrates that a sufficient condition to decouple the design of $\mathbf{A}(\omega, p, \phi)$ and $\mathbf{h}(\omega, \beta)$ is to steer the global and local filters towards the same angle. 


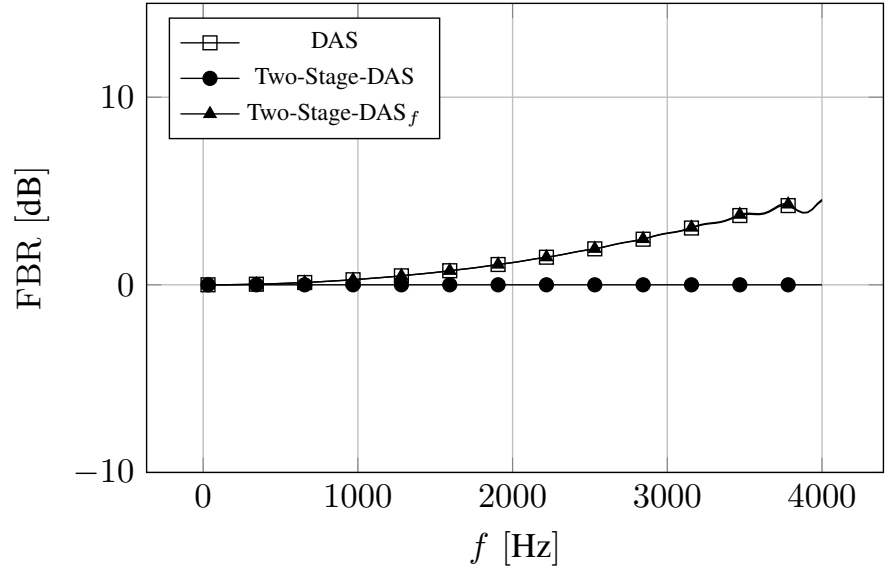

(a)

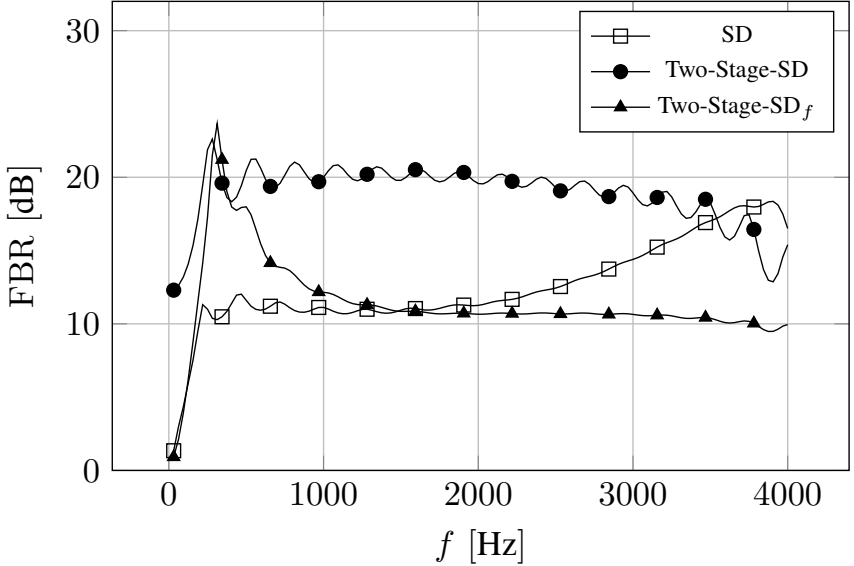

(b)

Fig. 8: Comparison in terms of FBR. In Fig. 8a the DAS is compared with the Two-Stage-DAS and the Two-Stage-DAS $f$. The Two-StageDAS uses a frequency-independent beampattern shape parameter while the Two-Stage-DAS $f$ a frequency-dependent one. In Fig. $8 \mathrm{~b}$ the SD

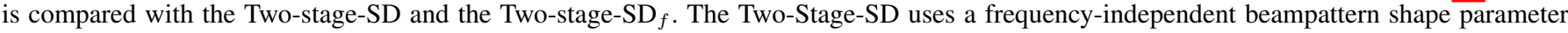
while the Two-Stage-SD $f$ a frequency-dependent one.

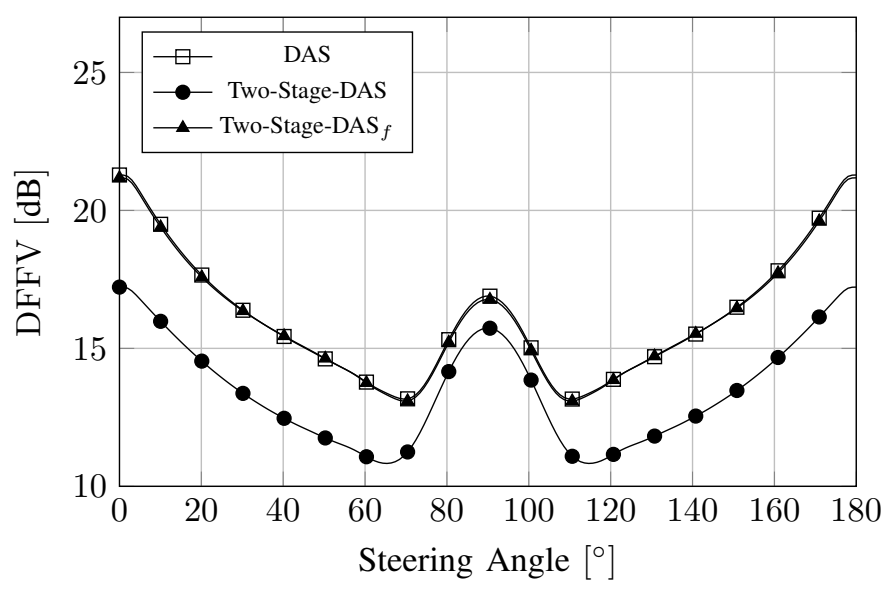

(a)

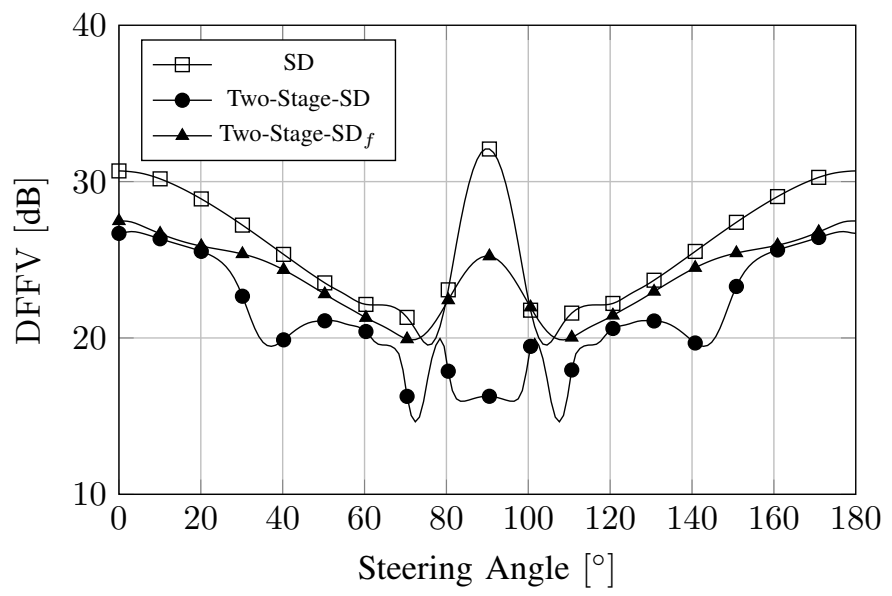

(b)

Fig. 9: Comparison in terms of Directivity Factor Frequency Variance (DFFV). In Fig. 9a the DAS is compared with the Two-Stage-DAS and the Two-Stage-DAS ${ }_{f}$. The Two-Stage-DAS uses a frequency-independent beampattern shape parameter while the Two-Stage-DAS $f$ a frequency-dependent one. In Fig. $9 \mathrm{~b}$ the SD is compared with the Two-stage-SD and the Two-stage-SD . The Two-Stage-SD uses a frequency-independent beampattern shape parameter, while the Two-Stage-SD $f$ a frequency-dependent one.

\section{REFERENCES}

[1] G. W. Elko and A. T. N. Pong, "A steerable and variable first order differential microphone array," in Proc. IEEE International Conference on Acoustics, Speech and Signal Processing (ICASSP), vol. 1, Apr. 1997.

[2] H. Teutsch and G. W. Elko, "First- and second- order adaptive differential microphone arrays," in Proc. IWAENC, Sep. 2001, pp. 35-38.

[3] M. Buck, "Aspects of first-order differential microphone arrays in the presence of sensor imperfections," European Transactions on Telecommunications, vol. 13, no. 1, pp. 115-122, Mar.-Apr. 2002.

[4] G. W. Elko, "Differential microphone arrays," in Audio Signal Processing for Next-Generation Multimedia Communication Systems, Y. Huang and J. Benesty, M. K. Norwell, Ed., 2004.

[5] J. Benesty and J. Chen, Study and Design of Differential Microphone Arrays, 1st ed., Springer, Ed., 2013.

[6] H. Zhang, J. Chen, and J. Benesty, "Study of nonuniform linear differential microphone arrays with the minimum-norm filter," Applied Acoustics, Elsevier, vol. 98, pp. 62-69, Nov 2015.

[7] J. Benesty, J. Chen, and I. Cohen, Design of Circular Differential Microphone Arrays, 1st ed., Springer, Ed., 2015.
[8] G. W. Elko, F. Pardo, D. Lopez, D. Bishop, and P. Gammel, "Surfacemicromachined mems microphone," in Proc. AES 115th Convention, Oct. 2003, pp. 1-8.

[9] G. W. Elko, "Steerable and variable first-order differential microphone array," Patent US 006041127 A, Mar. 21, 2000.

[10] E. De Sena, H. Hacihabiboglu, and Z. Cvetkovic, "On the design and implementation of higher order differential microphones," IEEE Transactions on Audio, Speech, and Language Processing, vol. 20, no. 1, pp. 162-174, January 2012.

[11] G. Huang, J. Benesty, and J. Chen, "Design of robust concentric circular differential microphone arrays," The Journal of the Acoustical Society of America, vol. 141, no. 5, pp. 3236-3249, 2017.

[12] L. Zhao, J. Benesty, and J. Chen, "Design of robust differential microphone arrays," IEEE/ACM Transactions on Audio, Speech, and Language Processing, vol. 22, no. 10, pp. 1455-1466, Oct. 2014.

[13] _ _ "Design of robust differential microphone arrays with the Jacobi - Anger expansion," Applied Acoustics, vol. 110, pp. 194 - 206, 2016.

[14] Y. Buchris, I. Cohen, and J. Benesty, "First-order differential microphone arrays from a time-domain broadband perspective," in 2016 IEEE International Workshop on Acoustic Signal Enhancement (IWAENC), 


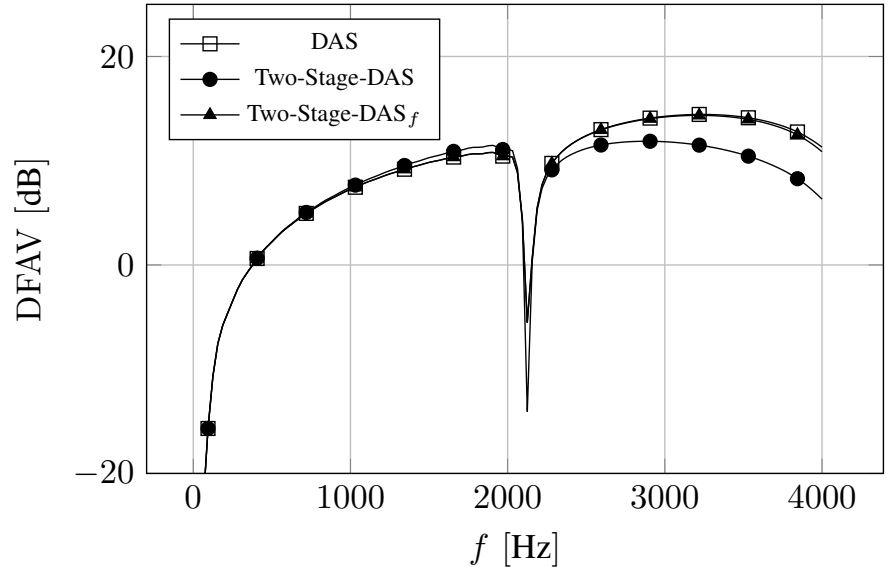

(a)

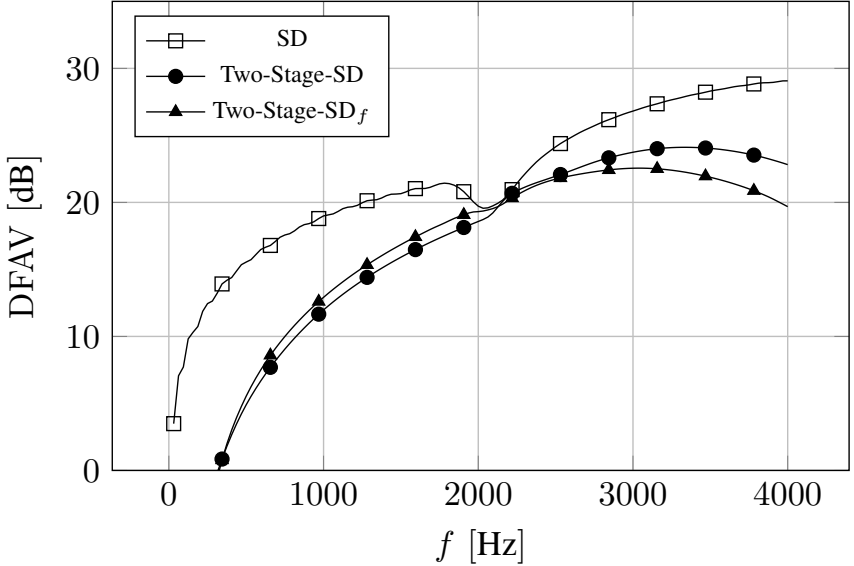

(b)

Fig. 10: Directivity Factor Angle Variance (DFAV). In Fig. 10a the DAS is compared with the Two-Stage-DAS and the Two-Stage-DAS . The Two-Stage-DAS uses a frequency-independent beampattern shape parameter while the Two-Stage-DAS $f$ a frequency-dependent one. In

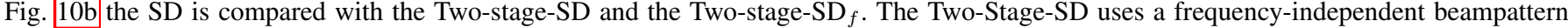
shape parameter while the Two-Stage-SD $f$ a frequency-dependent one.

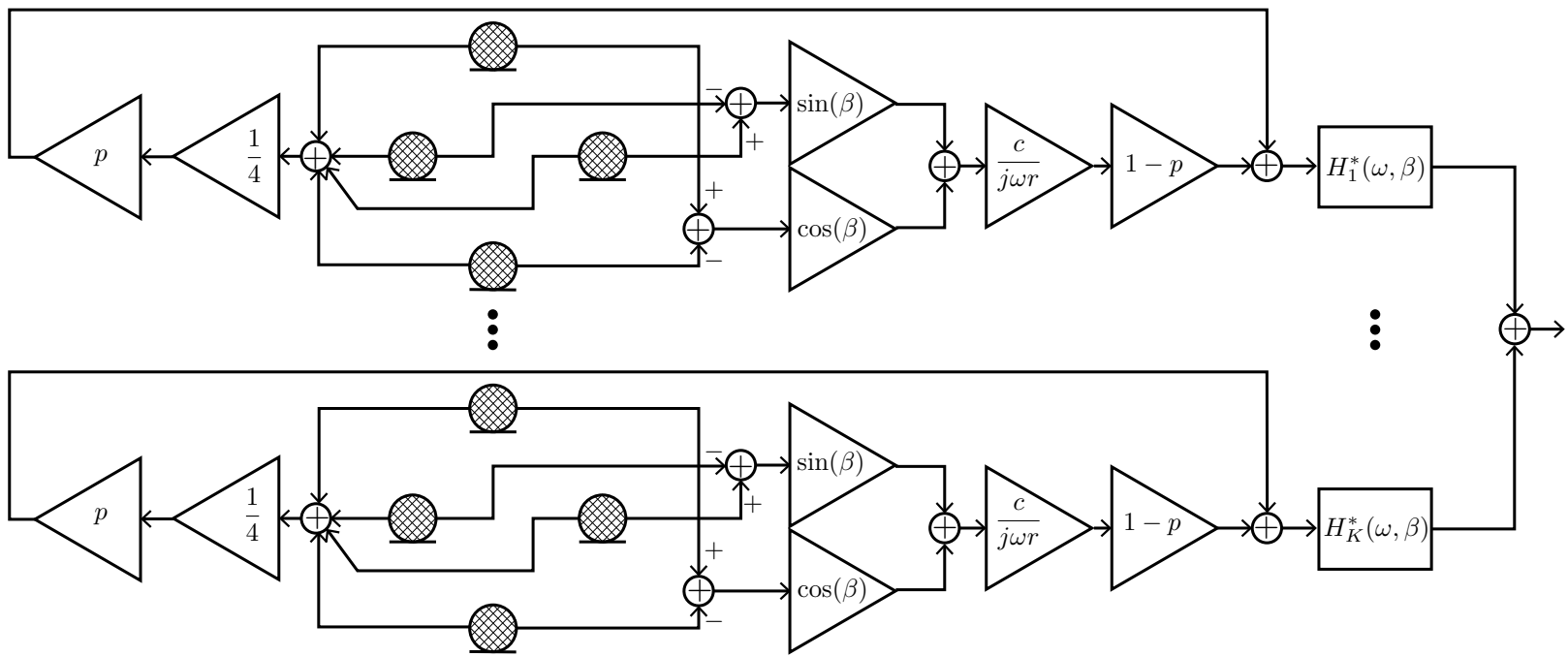

Fig. 11: Straightforward implementation of the proposed system.

Sept 2016, pp. 1-5.

[15] A. Bernardini, F. Antonacci, and A. Sarti, "Wave digital implementation of robust first-order differential microphone arrays," IEEE Signal Processing Letters, vol. 25, no. 2, pp. 253-257, Feb 2018.

[16] A. Bernardini, M. D'Aria, R. Sannino, and A. Sarti, "Efficient continuous beam steering for planar arrays of differential microphones," IEEE Signal Processing Letters, vol. 24, no. 6, pp. 794-798, June 2017.

[17] A. Bernardini, M. D'Aria, and R. Sannino, "Beamforming method based on arrays of microphones and corresponding apparatus," Patent US 20170374454 A1, Dec. 28, 2017.

[18] G. Huang, J. Benesty, and J. Chen, "On the design of frequency-invariant beampatterns with uniform circular microphone arrays," IEEE/ACM Transactions on Audio, Speech, and Language Processing, vol. 25, no. 5, pp. 1140-1153, May 2017.

[19] X. Wu and H. Chen, "Design and analysis of second-order steerable differential microphone arrays," in 2017 25th European Signal Processing Conference (EUSIPCO), Aug 2017, pp. 1245-1249.

[20] J. Byun, Y.-c. Park, and S. W. Park, "Continuously steerable secondorder differential microphone arrays," The Journal of the Acoustical Society of America, vol. 143, no. 3, pp. EL225-EL230, 2018.

[21] J. Lovatello, A. Bernardini, and A. Sarti, "Steerable circular differential microphone arrays," in 2018 26th European Signal Processing Conference (EUSIPCO), Sep. 2018, pp. 11-15.

[22] X. Wu, H. Chen, J. Zhou, and T. Guo, "Study of the mainlobe mis- orientation of the first-order steerable differential array in the presence of microphone gain and phase errors," IEEE Signal Processing Letters, vol. 21, no. 6, pp. 667-671, June 2014.

[23] X. Wu and H. Chen, "Directivity factors of the first-order steerable differential array with microphone mismatches: Deterministic and worstcase analysis," IEEE/ACM Transactions on Audio, Speech, and Language Processing, vol. 24, no. 2, pp. 300-315, Feb 2016.

[24] M. Brandstein and D. Ward, Microphone arrays: signal processing techniques and applications. Springer Science \& Business Media, 2013.

[25] P. Stoica and R. Moses, Spectral Analysis of Signals. Upper Saddle River, NJ, USA: Prentice Hall, 2004.

[26] H. Cox, R. Zeskind, and T. Kooij, "Practical supergain," IEEE Transactions on Acoustics, Speech, and Signal Processing, vol. 34, no. 3, pp. 393-398, June 1986.

[27] H. Cox, R. Zeskind, and M. Owen, "Robust adaptive beamforming," IEEE Transactions on Acoustics, Speech, and Signal Processing, vol. 35, no. 10, pp. 1365-1376, October 1987.

[28] R. Berkun, I. Cohen, and J. Benesty, "Combined beamformers for robust broadband regularized superdirective beamforming," IEEE/ACM Transactions on Audio, Speech and Language Processing (TASLP), vol. 23, no. 5, pp. 877-886, 2015.

[29] R. M. M. Derkx and K. Janse, "Theoretical analysis of a first-order azimuth-steerable superdirective microphone array," IEEE Transactions on Audio, Speech, and Language Processing, vol. 17, no. 1, pp. 150- 


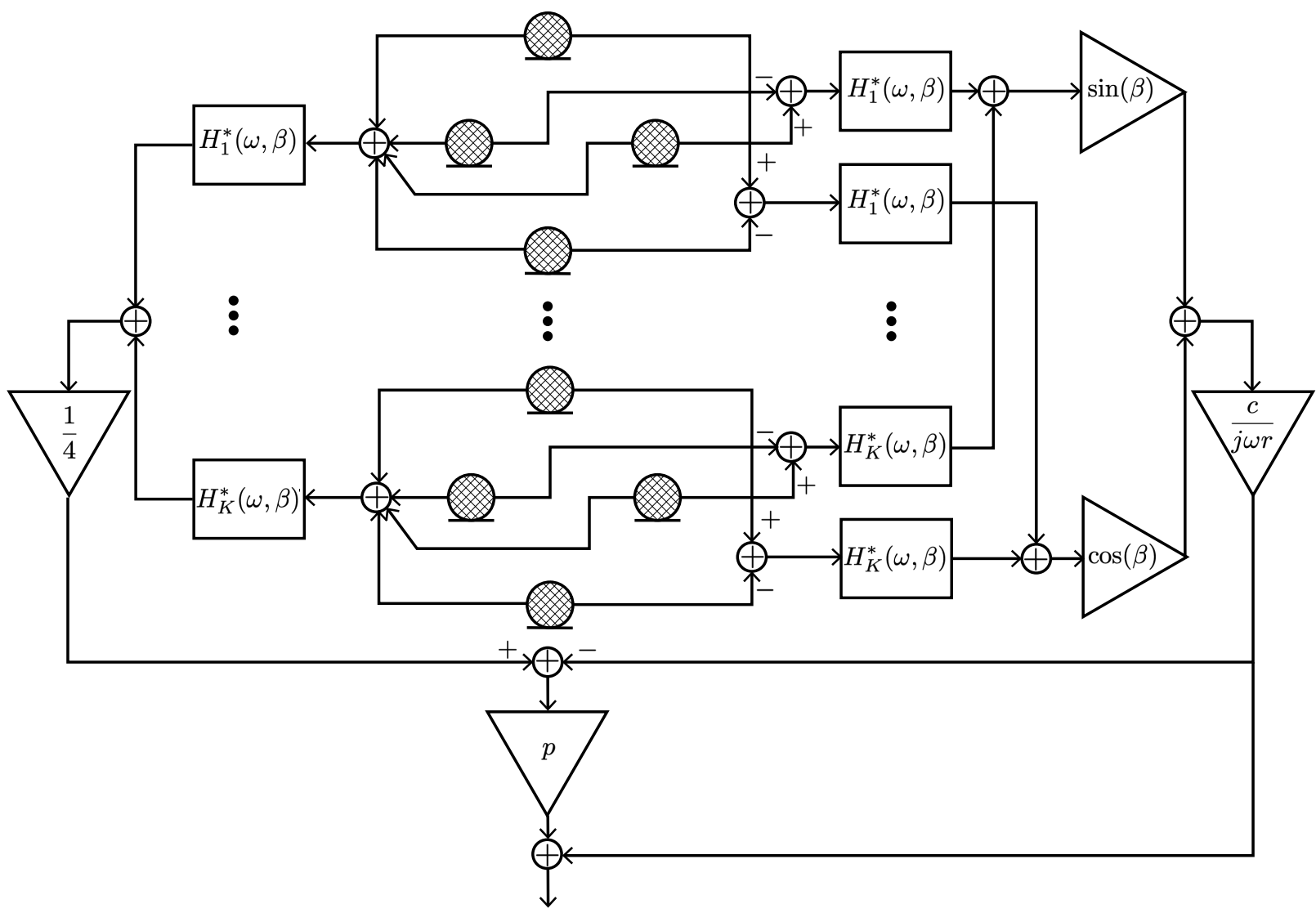

Fig. 12: Modified implementation of the proposed system that allows us to perform the "morphing" between a DAS-like beamformer and a SD-like beamformer in an efficient fashion.

162, Jan 2009.

[30] A. Bjorck, Numerical methods for least squares problems. Siam, 1996, vol. 51.

[31] M. Bydder, "Solution of a complex least squares problem with constrained phase," Linear algebra and its applications, vol. 433, no. 11-12, pp. 1719-1721, 2010.

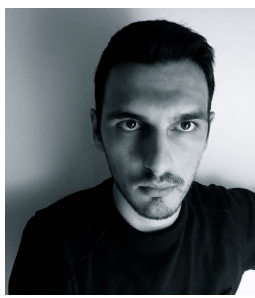

Federico Borra (S'17) received the B.S. degree, in 2014, and the M.S. degree (cum laude), in 2016, in computer engineering from the Politecnico di Milano, Italy, where he is currently pursuing the $\mathrm{Ph} . \mathrm{D}$. degree in information engineering with the Dipartimento di Elettronica, Informazione and Bioingegneria. His main research concerns spacetime audio signal processing.

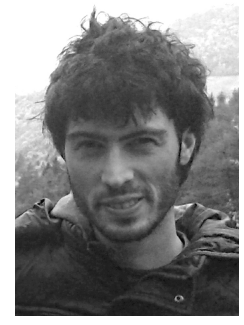

Alberto Bernardini (S'16-M'19) received his B.S. degree from the University of Bologna, Italy, in 2012 and his M.S. degree (cum laude) from the Politecnico di Milano, Italy, in 2015, both in Computer Engineering. In 2019, he received his Ph.D. degree (cum laude) in Information Engineering from the Politecnico di Milano, Italy, where he is currently a postdoctoral researcher. His main research interests are audio signal processing and modeling of nonlinear systems. He authored about 20 publications in international journals and proceedings of international conferences. He is also the first author of an international patent.

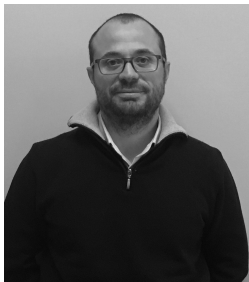

Fabio Antonacci (M'14) was born in Bari, Italy, on July 26, 1979. He received the Laurea degree in 2004 in telecommunication engineering and the $\mathrm{Ph} . \mathrm{D}$. degree in information engineering in 2008, both from the Politecnico di Milano, Milan, Italy. $\mathrm{He}$ is currently an Assistant Professor at the Politecnico di Milano. His research focuses on spacetime processing of audio signals, for both speaker and microphone arrays (source localization, acoustic scene analysis, rendering of spatial sound) and on modeling of acoustic propagation. He is a member of the IEEE Audio and Acoustic Signal Processing Technical Committee and of the EURASIP SAT on Audio, Speech and Music Signal Processing. 


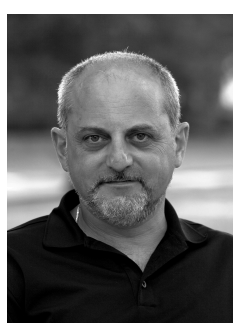

Augusto Sarti (M'04-SM'13) received the M.S. and $\mathrm{Ph} . \mathrm{D}$. degrees in electronics and information engineering from the University of Padua, Italy, in 1988 and 1993, respectively. In 1993, he joined the Faculty of the Politecnico di Milano, Italy, where he is currently a Full Professor. In 2013, he also joined the University of California, Davis. His research interests are in the area of multimedia signal processing, with a particular focus on sound analysis, synthesis and processing, computational acoustics, and music processing. He has co-authored over 250 scientific publications on international journals and congresses and holds numerous patents in the multimedia signal processing area. He coordinates the activities of the Musical Acoustics Lab and the Sound and Music Computing Lab of the Politecnico di Milano. He has been the promoter/coordinator and/or contributor to numerous (20+) European projects. He is an active member of the IEEE Technical Committee on Audio and Acoustics Signal Processing. $\mathrm{He}$ is on the Editorial Board of the IEEE. 